\title{
Analysis of the Acting Forces in a Theory of Catalysis and Mechanochemistry
}

\author{
Wolfgang Quapp, ${ }^{* \dagger}$ Josep Maria Bofill, $^{*,+}$ and Jordi Ribas-Ariño*,II \\ Mathematisches Institut, Universität Leipzig, PF 100920, D-04009 Leipzig, Germany, \\ Departament de Química Orgànica, Universitat de Barcelona, and Institut de Química Teòrica i \\ Computacional, Universitat de Barcelona, (IQTCUB), Martí i Franquès, 1, 08028 Barcelona \\ Spain, and Departament de Ciència de Materials i Química Física, Secció de Química Física \\ and IQTCUB, Universitat de Barcelona, Martí i Franquès, 1, 08028 Barcelona Spain \\ E-mail: quapp@uni-leipzig.de; jmbofill@ub.edu; j.ribas@ub.edu
}

date: March 21, 2017

R2 submitted: J.Phys.Chem.A

\begin{abstract}
The theoretical description of a chemical process resulting from the application of mechanical or catalytical stress to a molecule is performed by the generation of an effective potential energy surface (PES). Changes for minima and saddle points by the stress are described by Newton trajectories (NTs) on the original PES. From the analysis of the acting forces we postulate the existence of pulling corridors build by families of NTs which connect the same stationary points. For different exit saddles of different height we discuss the corresponding pulling corridors; mainly by simple two-dimensional surfaces models. If there are different exit saddles then there can exist saddles of index two, at least, in between. Then the case that

\footnotetext{
*To whom correspondence should be addressed

$\dagger$ Leipzig University

Universitat de Barcelona

IIUniversitat de Barcelona
} 
a full pulling corridor crosses a saddle of index two, is the normal case. It leads to an intrinsic hysteresis of such pullings for the forward or the backward reaction. Assuming such relations we can explain some results in the literature. A new finding is the existence of roundabout corridors which can switch between different saddle points by a reversion of the direction. The findings concern the mechanochemistry of molecular systems under a mechanical load as well as the electrostatic force and can be extended to catalytic and enzymatic accelerated reactions. The basic and ground ansatz includes both kinds of forces in a natural way without an extra modification.

\section{INTRODUCTION}

The application of mechanical stress to molecular systems has recently attracted significant interest as a means of controlling chemical reactions. Under stress the minimums and saddle points (SP) of the potential energy surface (PES) change their relations. This concerns the energy height as well as the molecular geometry. Curves for such changes in the configuration space of a molecule are the aim of this paper. In theoretical chemistry a basic concept already exists underlying many widely used models, namely, the reaction path (RP). The RP is a one-dimensional description of a chemical reaction through a sequence of molecular geometries in an $N$-dimensional configuration space. A reaction mechanism is described through the PES model by means of RPs. We use $N=3 n-6$ for the number of non-redundant internal coordinates, $\mathbf{r}$, and $n$ is the number of the atoms of the molecular system. Such a simplified one-dimensional description generally makes it possible to move on the PES. A well known type of RP models is the Newton trajectory (NT). ${ }^{1-8}$ For this type of RP holds that at every point of the curve the gradient of the PES points into the same direction, a direction of a prescribed search vector. On the other hand, this property is the central idea of models of mechanochemical stress applied to a molecular system where the search direction is now the direction of the stress vector. This is the reason why NTs should be taken into account as the basic models of a great variety of mechanochemical problems. ${ }^{9}$ The present study is a follow-up of a first investigation of the realm of mechanochemistry with the NTs tool. ${ }^{10,11}$ 
In the last years, the phenomena of the action of a mechanical stress over a molecular system have motivated experimental and theoretical researches (see Refs. 12-23 and references therein) We will here quantify molecular stress geometrically. Basically, the model employed to rationalize mechanochemical experiments was created early by Eyring et al., ${ }^{24}$ by Bell ${ }^{25}$ in 1978, and it was used, for example by Bustamante et al. ${ }^{26}$ in 2004. More recently, it was recreated and treated by Ong et al., ${ }^{27}$ by Ribas-Ariño et al. ${ }^{28}$ and by Wolinski and Baker ${ }^{29}$ in the same year 2009. The generally accepted model ${ }^{15,19,30}$ consists in a first order perturbation on the associated PES of the unperturbed molecular system, $V(\mathbf{r})$, due to a stress or pulling force vector, $\mathbf{f}$, by

$$
V_{f}(\mathbf{r})=V(\mathbf{r})-\mathbf{f}^{T} \cdot\left(\mathbf{r}-\mathbf{r}_{o}\right)
$$

where the symbol $T$ means transposition of a vector or a matrix. $\mathbf{r}$ is the coordinate vector, $\mathbf{r}_{o}$ is any fixpoint, and $\mathbf{f}$ is the force vector. In this equation, being the starting point for the theory of mechanochemistry, the scalar product $\sum_{i}^{N} f_{i}\left(r_{i}-r_{o i}\right)$ with the force vector, f, ensures that only the part of the coordinate vector, $\left(\mathbf{r}-\mathbf{r}_{o}\right)$, in the direction of $\mathbf{f}$ acts. The subtrahend in the equation is a hyperplane in the space $\mathbf{I R}^{N+1}$ whith slope in the direction of the force, $\mathbf{f} \in \mathbf{R}^{N} . V_{f}$ is named the force-transformed PES, ${ }^{15,28,31,32}$ or the effective PES. The displacement of the stationary points of the new effective PES is described by NTs. ${ }^{9-11}$ The force, $\mathbf{f}$, can be a pull or a push. It is worth mentioning that a similar model was discussed by Thornton ${ }^{33}$ for the prediction of the effect of substituent changes on the transition state (TS) geometry.

The formalism employed to study mechanochemistry can also be applied to explore how external electric fields can catalyze and control reactions ${ }^{34-36}$ when these fields are constant in time and position. ${ }^{34}$ One could even think of using this formalism as a first-order approximation to study catalytic and enzymatic processes. Notice that in the present model, the direction and the magnitude of $\mathbf{f}$ are constant. (Note that also non-constant forces are studied in the past. ${ }^{37-39}$ ) The used constant force here is certainly a strong approximation, but it might be useful when it comes to understand and design catalysts and enzymes. In this case, the direction and the magnitude of 
vector $\mathbf{f}$ are fixed for every special catalyst. Indeed, if we can quantify all the electrostatic and nonelectrostatic parts of the action of a catalyst (an enzyme in biochemistry), into a force represented mathematically by a vector, $\mathbf{f}$, then we can handle the change of the PES by the ansatz of Eq. (1). The action of the catalyst will then be explicitly calculable and interpretable by the displacement of a TS and a reactant minimum of the original PES, V(r), to the effective PES, $V_{f}(\mathbf{r})$.

In the present context it is important to consider that historically the problem of enzyme catalysis was discussed early by Haldane ${ }^{40}$ and Pauling. ${ }^{41}$ There should be an 'active region of the surface of the enzyme which is closely complementary in structure not to the substrate molecule itself, ..., but rather to the substrate molecule in a strained configuration..., meaning the transition state (TS). '... and caused by the forces of attraction to assume the strained state which favors the chemical reaction... ${ }^{41}$ Thus Pauling proposed that the enzyme binds the TS. It was named TS stabilization (TSS), in contrast to reactant destabilization. ${ }^{42-44}$ The activation energy is lowered for the catalyzed versus the uncatalyzed process, giving rise to large rate accelerations. It is equivalent to the action of Eq. (1) for pulling which leads us to the remark that this equation not only describes the pulling process, but can generally describe catalysis as well. ${ }^{10,11,26}$

A second look back has to appreciate the early guess of Warshel ${ }^{42,45-47}$ that the catalytic effect of enzymes has an electrostatic origin. An electrostatic force of the enzyme stabilizes the TS of the accelerated reaction: this expresses in words what Eq. (1) expresses as a linear ansatz by a formula. Maybe this ansatz is too simple for a given problem, but it explains many observed and studied results and now remains the task to determinate the force, $\mathbf{f}$, for every enzyme. The methodology reported in the next section allows us to calculate explicitly the lowering of the activation energy by the enzyme, if $\mathbf{f}$ is known (and if we can calculate, or approximate the PES, or the free energy surface of the reaction under consideration).

At this point, it should be mentioned that mechanochemistry and catalysis should not be considered as two different facets of chemistry under the point of view of Eq. (1). Indeed, a direct combination of electrostatical and mechanical forces is found in molecular motors. These two facets also meet in the research area of mechanical activation of catalysts ${ }^{48,49}$ and on experiments 
in which an enzyme acts on a substrate subject to tensile stress. ${ }^{50,51}$

In this article, we present the chemical consequences of a topological study of generic PESs based on NTs that are related with the basic ansatz Eq. (1). For this reason, the conclusions drawn are completely general, independent of the type of quantum mechanical calculation of the PES. Nevertheless, chemical examples from the literature are discussed under this new view corroborating the present theory. Our analysis demonstrates the existence of streams of NTs (which will be referred to as chemical corridors) that are crucial to understand and model the acting forces in mechanochemistry and catalytic phenomena. We will unveil the unforeseen challenges and complexity embodied in Eq. (1). The article is organized as follows. Next we explain the theory of NTs for the understanding of a chemical pulling/pushing process, or an action of a catalyst (enzyme). This theoretical part is a summary of that published in references 9,10, and is briefly exposed and explained, in this way the article is self-contained. Then we use two-dimensional test surfaces to explain the impact of stress on the topography and the stationary points of the effective PES. We mainly treat the 'strong multidimensional case' ${ }^{22}$ with competing reaction pathways. We show that already for two dimensions, if at least two competing RPs exist, we can find rather complicated areas for the movement of the stationary points on the PES under stress. Nevertheless, though pure theoretical, these generic surfaces are taken from well tested models of specific chemical systems. The paper presents at the end a Discussion, and some Remarks with a Conclusion.

\section{METHODOLOGY}

For a given force, $\mathbf{f}$, we have an effective PES, $V_{f}(\mathbf{r})$, which has a new inherent chemistry with respect to the unperturbed PES, $V(\mathbf{r})$, such as other reaction rates and other chemical properties. Of course, the linear perturbation in Eq. (1) is the simplest model. ${ }^{52}$ Meanwhile, time was used to derive new basic ideas of mechanochemistry. ${ }^{15,52-57}$ The force $\mathbf{f}$ in Eq. (1) may be determined by

the change of a distance between two pulling points of the molecule, ${ }^{58}$ or by any other experimental setup. Formerly it was associated with one of the $N=3 n-6$ internal coordinates ${ }^{58}$ or a linear 
combination of them. ${ }^{27}$ Throughout the present work we assume for simplicity that the plane of two intrinsic coordinates $(x, y)$ is the stage where the pulling works. These coordinates may describe the weakest point of a molecule where it breaks preferentially. ${ }^{59}$ We note that the theory and the use of NTs can be applied to $N$-dimensional systems with any large $n$ for the number of included atoms.

Due to the external force, the stationary points are located at different positions on the effective potential, $V_{f}(\mathbf{r}),{ }^{56}$ with respect to the unperturbed potential, $V(\mathbf{r})$, where it holds $\nabla_{\mathbf{r}} V(\mathbf{r})=\mathbf{g}(\mathbf{r})=$ 0. $\mathbf{g}(\mathbf{r})$ is the gradient of the unperturbed PES. The stationary points on the effective potential have to satisfy the analogous condition, $\nabla_{\mathbf{r}} V_{f}(\mathbf{r})=\mathbf{0}$. Since $V_{f}(\mathbf{r})$ is the one given in Eq. (1) there follows that the new minimums or SPs should satisfy

$$
\nabla_{\mathbf{r}} V_{f}(\mathbf{r})=\mathbf{0}=\mathbf{g}-\mathbf{f}
$$

One searches a point where the gradient of the unperturbed PES, $\mathbf{g}$, has to be equal to the mechanochemical force, $\mathbf{f}$, being the force that induces the chemical process. If the mechanical stress in a defined direction is $\mathbf{f}=F \mathbf{l}$ with a fixed unit vector, $\mathbf{l}$, then it is $\mathbf{l}=\mathbf{g} /|\mathbf{g}|$ and $F=|\mathbf{g}|$ is the magnitude at the stationary points since from Eq. (2) we have, $\mathbf{0}=\mathbf{l}(|\mathbf{g}|-F)$, being satisfied if the former equality is also satisfied. Eq. (2) means that the tangential hyperplane to the original PES, characterized by the gradient, $\mathbf{g}$, is equal to the hyperplane of the pulling force, $\mathbf{f}^{T} \mathbf{r}$, in Eq. (1). The case $F=0$ is named the pure thermal limit. ${ }^{15}$ This is the case without a mechanical load.

Now we treat a fixed direction of $\mathbf{l}$, but different magnitudes of the forces, $F$. For changing magnitudes, $F$, we get a series of effective PESs (1), and on every effective PES we get its stationary points. So we get a path following for the curve of the force displaced stationary points (FDSPs). ${ }^{30}$ The minimums and the SPs of any index of the effective PES (1) change and they are on the FDSPs curve, because at these points holds $\pm|\mathbf{g}|=F$. A FDSPs is the path connecting the stationary states of the perturbed PESs. Each effective PES of this ensemble corresponds to a given 
magnitude $F$ of the force but to the same direction $l$ of application.

The FDSPs are on the solution of the differential equation of Branin ${ }^{60}$ which we can use in $N=3 n-6$ nonredundant coordinates 9,10

$$
\frac{d \mathbf{r}}{d t}= \pm \mathbf{A}(\mathbf{r}) \mathbf{g}(\mathbf{r})
$$

$t$ is a curve parameter and the matrix $\mathbf{A}$ is constructed as a product of the determinant of the Hessian, $\mathbf{H}$, of the unperturbed PES with the inverse Hessian,

$$
\mathbf{A}=\operatorname{Det}(\mathbf{H}) \mathbf{H}^{-1}
$$

At the given g, the Branin equation yields a "Newton Trajectory" (NT) corresponding to the FDSPs for this $\mathbf{g}$. The Branin equation is nothing but a simple strategy to generate the points on the FDSPs without solving $\mathbf{g} /|\mathbf{g}|=\mathbf{l}$ and $\pm|\mathbf{g}|=F$ for several values of the force magnitude.

The signs ' \pm ' in Eq. (3) are used to allow the curve to go uphill from a minimum, or downhill from an SP. Curves $\mathbf{r}(t)$ satisfying this expression are called Newton trajectories (NT). A property of Eq. (3) is that the gradient at every curve point always points into the same direction, $\mathbf{l}$, if it had this direction at an initial point. Then holds the parallelity of the vectors, $\mathbf{l}|| \mathbf{g}$, throughout the path. ${ }^{1,2}$ Note that different directions $\mathbf{l}$ cause a spread of NTs, as it will appear in the various examples later in the paper.

The solution curve of the Branin equation is a regular curve from a point near the minimum to an SP if no valley-ridge inflection (VRI) point is crossed. ${ }^{2}$ However, if this curve crosses in its evolution a VRI point, then this NT curve is named a singular NT. It bifurcates at the VRI. A VRI point is a region on the PES where the curvature orthogonal to the gradient becomes zero. There usually a valley or a ridge bifurcate. ${ }^{2,61}$ The Branin Eq. (3) is a well-known model for RPs. ${ }^{1,2,5,8}$ This RP model is especially used here for the FDSPs: for every special magnitude of the force, $F$, we get a moved stationary point of the new effective potential, $V_{f}(\mathbf{r})$ with $\mathbf{f}=F \mathbf{l}$ and $\mathbf{g}=\mathbf{l}|\mathbf{g}|$, but these points can be represented on the NT of the original PES, $V(\mathbf{r})$, where this NT is used for the 
pulling direction. The reason is that this special pulling direction does not change the description of this special NT on every effective PES under the pulling.

Note that the NT which describes the curve of FDSPs is not used here for a reaction path. Nevertheless, the effective stationary points of $V_{f}(\mathbf{r})$ of Eq. (1) can be connected by a chemical reaction path to describe the usual reaction process over the effective SP. That can be an NT, or the IRC, ${ }^{62}$ or any other definition. That path is named "mechanical coordinate" 26 but we do not treat it here.

If one moves on the path of FDSPs one moves, so to say, from one force displaced effective PES to the next. Step by step one has to increase the norm of the force, $F$, beginning at the original stationary points with $F=0$ in the thermal limit: there is a part of the pathway from the minimum uphill, and a part leading usually downhill from the SP. If the force increases further and further, the two parts of the FDSPs have to meet. Here the norm of the gradient has its maximum. The NT goes through a shoulder on the effective PES. The curvature of the PES along the corresponding NT is zero, because at the meeting point of the effective minimum and the effective SP we have $d V_{f}(\mathbf{r}) / d t=\mathbf{l}^{T} \mathbf{A l}(|\mathbf{g}|-F)|\mathbf{g}|=0$. As a consequence, the barrier of the original PES disappears here. In other words, on the effective PES, $V_{f}(\mathbf{r})$, with the maximal rupture force, ${ }^{63}$ the SP disappears, and the pulling force realizes the reaction. (This may happen somewhat earlier because of the existence of the zero point energy at the former minimum.) We propose to name the point the barrier breakdown point (BBP). Its necessary mathematical formula is 10,64

$$
\operatorname{Det}(\mathbf{H})=\mathbf{0} \text {. }
$$

Note that $\mathbf{H}$ is always the Hessian of the original zero-force PES, $V(\mathbf{r})$, because the model Eq. (1) and Eq. (2) do not influence the calculation of the Hessian ${ }^{33}$ (the first order perturbation in ansatz (1) does not change the second derivatives of the PES because $\mathbf{f}$ is constant and consequently independent of $\mathbf{r}$, differentiating Eq. (1) two times with respect to $\mathbf{r}$ we have $\nabla_{\mathbf{r}} \nabla_{\mathbf{r}}^{T} V_{f}(\mathbf{r})=\nabla_{\mathbf{r}} \nabla_{\mathbf{r}}^{T} V(\mathbf{r})=$ $\mathbf{H}(\mathbf{r})$, thus the Hessian coincides for both PESs). The idea of the proof of condition Eq. (5) is that 
the BBP is a turning point of the function $|\mathbf{g}|$ along the NT. A proof of Eq. (5) is given in Ref. 10. The definition of an optimal pulling direction ${ }^{10}$ is also derived: the mechanical force to be applied to the molecule of interest is $\mathbf{f}=F \mathbf{l}$ where $F=\sqrt{\mathbf{g}^{T} \mathbf{g}}$. To each NT belongs the corresponding lvector, and by varying it we have different NT curves. All the regular NTs, that leave the minimum and arrive at the same SP, cross at least once a $\operatorname{Det}(\mathbf{H})=0$-line. The $\operatorname{Det}(\mathbf{H})=0$-line that each NT crosses gives the $\mathrm{BBP}$ of this NT, the maximal rupture force, $F_{\max }$. This is due to the fact that at the BBP point of a regular NT, the gradient norm $|\mathbf{g}|$, takes its maximum value along this NT curve. If we compare all NTs of such a set, then the NT which gives the lowest value of $F_{\text {max }}$ is named the optimal NT. It coincides with a gradient extremal (GE) exactly at the intersection point with the $\operatorname{Det}(\mathbf{H})=$ 0-line. ${ }^{10}$ In this special BBP, the $\operatorname{Det}(\mathbf{H})=0$-line, the GE and the optimal regular NT meet. The optimal BBP is a stationary point on the function $|\mathbf{g}|$ crossed by the optimal NT. On higher dimensional PESs, the condition $\operatorname{Det}(\mathbf{H})=0$ may describe a manifold. So, the definition of optimality works in any dimension.

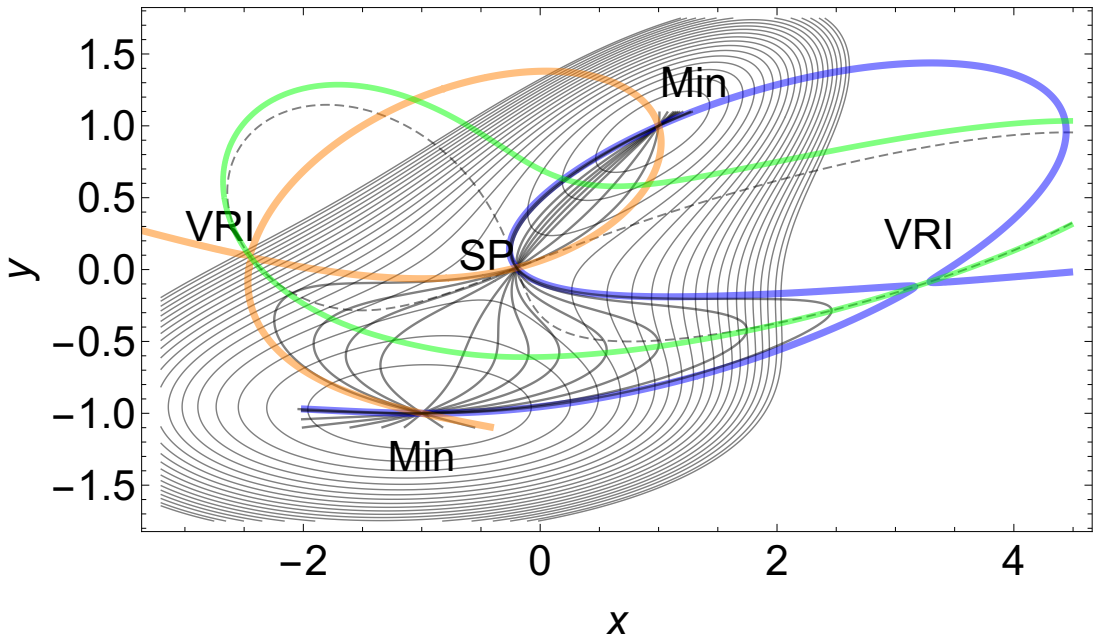

Figure 1: Direct chemical corridor of type 1 of the PES of Eq.(S1) in SI. All the regular NTs between the two singular NTs (in blue and orange color) form this corridor. Some regular NTs (in gray) are shown. The BBP condition, $\operatorname{Det}(\mathbf{H})=0$, is the green line. A singular NT is the Newton trajectory that passes through a VRI point, whereas a regular NT does not pass through this type of points. The spreading of pulling directions can be large. ${ }^{65}$ All NTs describe pulling directions to different families of effective PESs. The surface without load is shown in the background for comparison.

Historically, the BBP was named the breaking point distance (for single bonds), ${ }^{15,63,66}$ and 
the corresponding magnitude $F_{\max }$ was the rupture force. BBPs for bonds in diatomic molecules are calculated, ${ }^{15,66,67}$ as well as the BBPs for single bonds in some polyatomics. ${ }^{66,67}$ The onedimensional BBP-problem is treated by Freund as well. ${ }^{68}$ With the present model, both the BBP and $F_{\max }$ can be predicted for large molecules, thus in a higher dimension. From a mathematical point of view the BBP concept is strongly related with the Catastrophe Theory. ${ }^{69-71}$ From this view BBP represents a catastrophe of the PES function being unfolded by a force affecting through the

additional perturbation term, $\mathbf{f}^{T}\left(\mathbf{r}-\mathbf{r}_{0}\right)$, of Eq. (1). ${ }^{10}$ This gives us the general structure of all possible effective PESs with zero eigenvalue at this point. For this reason this mathematical model can be used to predict mechanochemistry mechanisms and catalysis. In the present study we will use corridors of NTs on test surfaces to treat regions of the PES where all NTs lead to the same stationary point. A pulling, or correspondingly a pushing along every of these NTs enforces the same type of FDSPs on the PES. We name such a common set of NTs a corridor, however, due to the physical-chemistry significance of this set of curves, we call it a chemical corridor. Of course, the BBPs of the NTs of a corridor can be very different in their energy height, as well as in their bond breaking gradient force. ${ }^{10,11}$

\section{MODELS FOR CHEMICAL CORRIDORS}

The theory herein presented aims to rationalize both mechanochemistry and catalysis. It is based on the topology or, more specifically, the topography of the common PES associated to different types of chemical mechanisms. In the following, we shall analyze the topography of the basic PES models, namely, (i) a PES describing an elementary pathway, (ii) a PES describing a process with two different reaction channels, and (iii) a PES describing a process with two different reaction channels that include intermediates. This will permit us to draw generic topological conclusions which are important for the general applicability of the present theory. 


\subsection{Potential energy surface with an elementary pathway}

We present as an example a simple PES with a one-step mechanism to analyze the nature of the corridors associated to this type of PES. As a generic representative of this class of PES, we use the skew double-minimum PES, ${ }^{6,10}$ whose mathematical description is given in the Supplementary Information (by Eq.(S1), the PES is shown in Figure 1). This PES has two minima, connected by one SP of index one (i.e, a transition state).

In Figure 1 the direct corridor between the two minima is represented by NTs. This kind of corridor is named of type 1. The green line is the BBP-condition $\operatorname{Det}(\mathbf{H})=0$. The dashed line is the convexity border of the PES given by $\mathbf{g}^{T} \mathbf{A} \mathbf{g}=0 .{ }^{72}$ The ridge and valley regions of the PES are separated by this line. Shown are the two singular NTs of the PES by blue and orange color. They bifurcate at two VRI points. They are the two border lines of the corridor. Thus a chemical corridor of type 1 is characterized by the set of regular NTs joining two minimums of the PES related with reactant and product. It goes through an SP of index 1 in between, and it is limited by singular NTs. Not all regular NTs of this corridor satisfy the reaction path requirement to rise up without a turning point (TP), but a subset of NTs do so. The regular NTs that belong to this subset are reaction pathways satisfying the condition of the minimum energy path. ${ }^{73}$ The gradient norm at the BBP line is the maximal force at which a reaction is enforced. ${ }^{26,65}$ Going along an RP the gradient norm achieves the maximum value at the intersection point with the BBP line. ${ }^{10}$ Of course, the maximal force significantly depends on the pulling direction.

The movement of the effective $\mathrm{SP}_{\text {eff }}$ is manifested in many enzymatic reactions. ${ }^{26,74}$ It was named stress-induced catalysis by the enzyme, in former treatments. However, we should note that inasmuch as the model of Eq. (1) fits to a reaction problem, the $\operatorname{Min}_{\text {eff }}$ moves also along the pulling NT. The two stationary points both move to come together at the BBP, at least. Depending on the PES, the BBP can be nearer to the original SP (late BBP or TSS) or to the original Min (early BBP). ${ }^{10}$ 


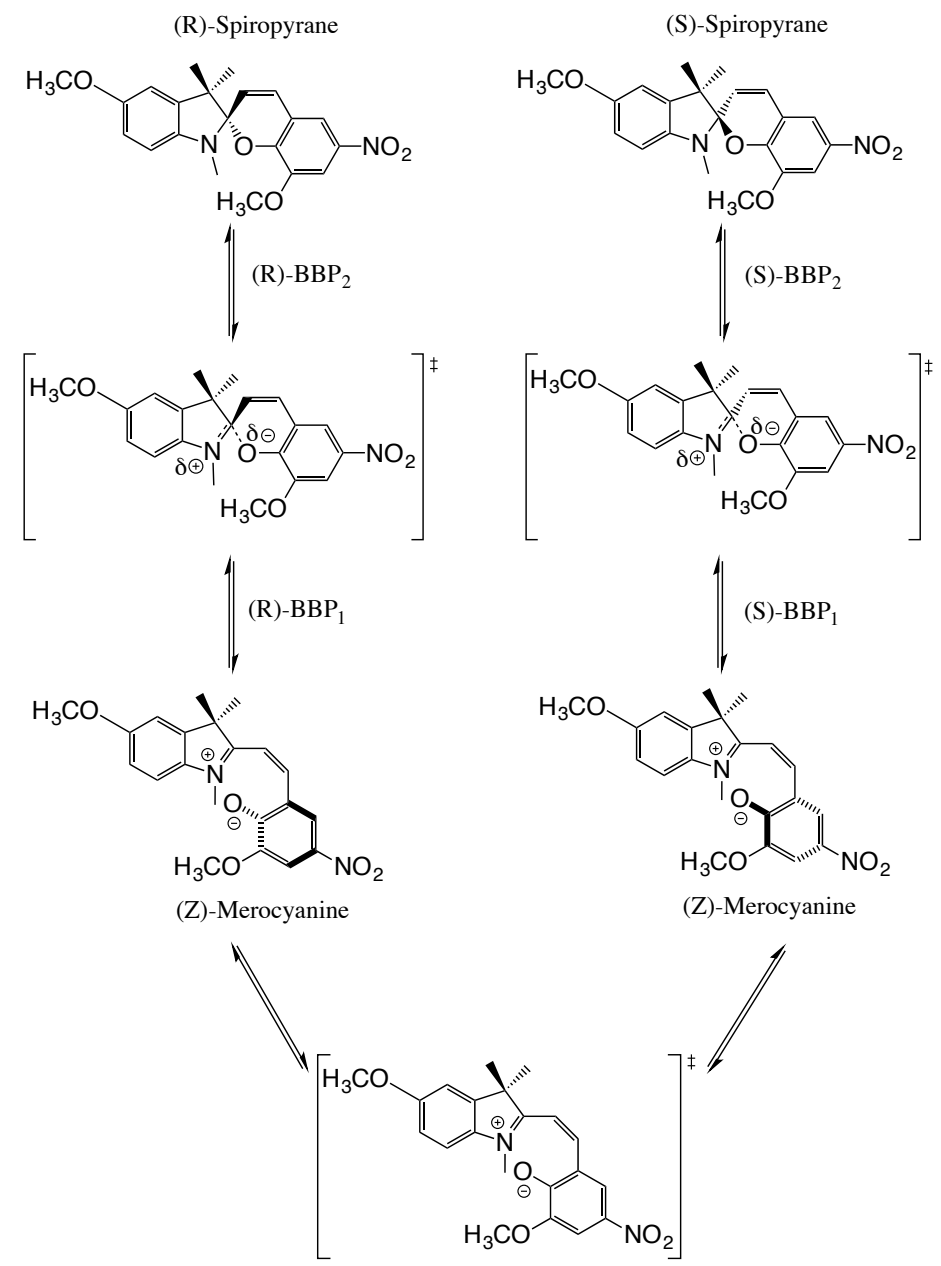

Figure 2: Schematic transformation of (Z)-Merocyanine to Spiropyrane. Depending on the conformational isomer of (Z)-Merocyanine, an enantiomer of Spiropyrane is obtained. The conformational interconversion of $(\mathrm{Z})$-Merocyanine takes place through a planar transition state indicated at the bottom.

Starting a pulling at the minimum at the point $(-1,-1)$ in Figure 1 , we may observe that the spreading of the tangent directions of the possible regular NTs is large. In contrast, starting a pulling at the minimum at $(1,1)$ in contrary directions, we can observe that the spreading of the tangent directions of the possible NTs is quite smaller. We recall that any NT of the corridor follows one and the same gradient direction through its evolution. Thus both starting points at the minimums encircle an equal range of gradient directions. This also happens at the common SP of index 1 which all NTs cross. From a chemical point of view, this topological conclusion means that if a given vectorial force is able to drive the system from reactants to products in a corridor of 
a)
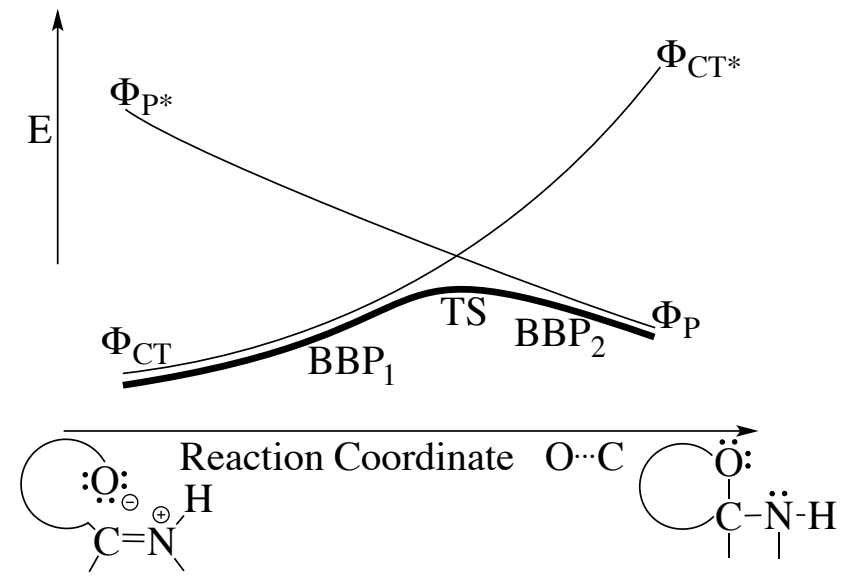

(Z)-Merocyanine Spiropyrane

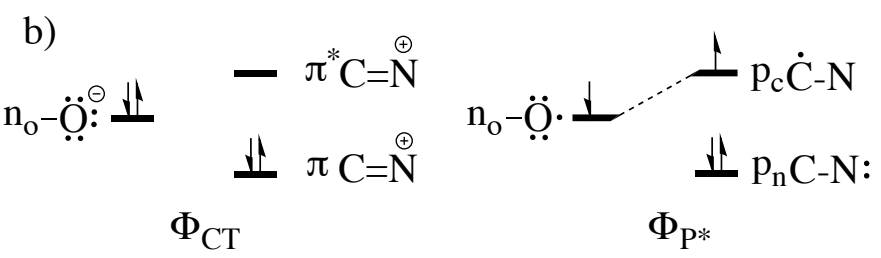

c)

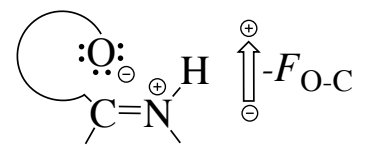

Figure 3: Transformation of (Z)-Merocyanine to Spiropyrane. a) Valence bond correlation diagram for the intramolecular reaction between the nucleophile $-\mathbf{O}^{-}$and the electrophile $>\mathbf{C}=\mathbf{N}^{+}<$. b) The charge transfer, $\Phi_{C T}$, and product, $\Phi_{P}$, valence-bond configurations projected from the molecular orbitals wave function for the $-\mathbf{O}^{-}$attack of a $>\mathbf{C}=\mathbf{N}^{+}<$bond. c) The bond breaking point $\mathrm{BBP}_{1}$ labeled according Figure $2 . \mathrm{BBP}_{1}$ has the major contribution of $\Phi_{C T}$, more than $\mathrm{BBP}_{2}$. Shown is the external electric field, $-\mathbf{F}_{\mathbf{O}-\mathbf{C}}$, along the "reaction coordinate" $(\mathbf{O} \cdots \mathbf{C})$.

type 1 , the reverse force will necessarily drive the system from products to reactants.

An example of this type of corridor within the context of mechanochemistry concerns the intramolecular addition of an electrophile to a nucleophile, such as the rearrangement of (Z)Merocyanine to Spiropyrane, see Figure 2. Each of the elementary pathways of Figure 2 (either the left-handside one or the right-handside one) corresponds to a type 1 corridor.In this particular example, we will consider that the PES is perturbed by an electric field rather than a mechanical force.

A valence bond diagram of this rearrangement is displayed in Figure 3. Panel (a) describes the 
a)

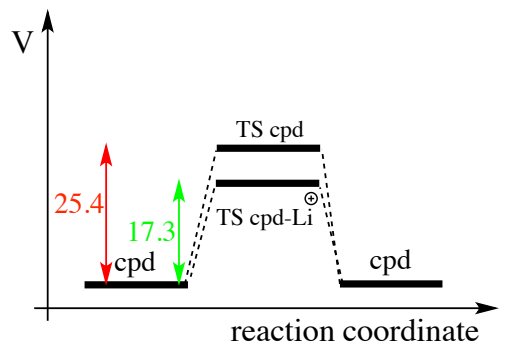

b)

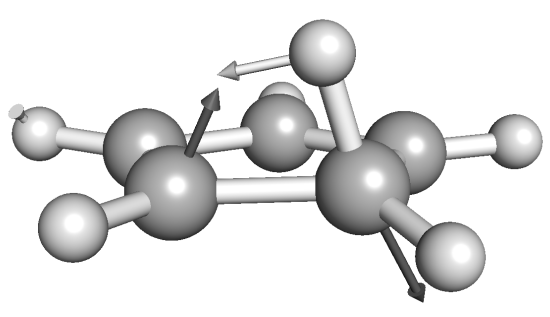

Figure 4: (a) Energy profile of the 1,2-sigmatropic H-shift rearrangement of cyclopentadiene without and with $\mathrm{Li}^{+}$as a catalyst. The energies which are given in $\mathrm{kcal} \mathrm{mol}^{-1}$ were computed at the MP2/6-31G* level of theory. (b) The BBP structure obtained from the IRC calculation. The arrows correspond to the components of the gradient vector at this point.

barrier formation and the TS electronic character. The rearrangement corresponds to a nucleophilic addition of the nucleophile $-\mathbf{O}^{-}$to an electrophile being an unsaturated group, $>\mathbf{C}=\mathbf{N}^{+}<$, where $\mathbf{N}$ is more electronegative than $\mathbf{C} .{ }^{75}$ The diagram involves two principal curves, which represent the (Z)-Merocyanine and the Spiropyrane states, $\Phi_{C T}$ and $\Phi_{P}$, respectively. Note that the (Z)Merocyanine state is charge transfer in nature. This charge transfer involves a single electron transfer from the nucleophile $-\mathbf{O}^{-}$to the electrophile group, $>\mathbf{C}=\mathbf{N}^{+}<$. The strongest mixing happens in the TS region, located at the crossing point between the electronic states. In panel (b) of Figure 3 is shown the charge transfer, $\Phi_{C T}$, and product, $\Phi_{P}$, valence-bond configurations which are projected from the molecular orbitals wave function for the $-\mathbf{O}^{-}$attack of a $>\mathbf{C}=\mathbf{N}^{+}<$bond. It is important to take into account that the $\Phi_{C T}$ state has a major contribution in the description of the electronic structure of $\mathrm{BBP}_{1}$, shown in panel (c) of Figure 3, in comparison to $\mathrm{BBP}_{2}$.

Let us deeper consider Figure 3 (a). When an external electric field is applied along the "reaction coordinate" $\mathbf{C} \cdots \mathbf{O}$, a negative $\mathbf{F}_{\mathbf{O}-\mathbf{C}}$ field stabilizes $\Phi_{C T}$, which shifts the TS towards the $\mathrm{BBP}_{2}$. Additionally, this stabilization of the $\Phi_{C T}$ state destabilizes the Spiropyrane resulting in a structural and energetic shift towards $\mathrm{BBP}_{2}$. The vector field, $-\mathbf{F}_{\mathbf{O}-\mathbf{C}}$, has the direction of the normalized gradient vector, $\mathbf{l}_{2}$ of the $\mathrm{BBP}_{2}$ being the optimal direction for pulling. Increasing the magnitude of the force, namely, $F_{\mathbf{O}-\mathbf{C}}$, both TS and Spiropyrane structures can coalesce at this 
BBP. The opposite effect for a positive vector field, $\mathbf{F}_{\mathbf{O}-\mathbf{C}}$, has the direction of the normalized gradient $\mathbf{l}_{1}$ of the $\mathrm{BBP}_{1}$. It is again optimal for pushing. It destabilizes the charge transfer state, $\Phi_{C T}$, which shifts the TS and the (Z)-Merocyanine toward $\mathrm{BBP}_{2}$. As in the previous case, increasing the magnitude of the force, the TS and (Z)-Merocyanine coalesce to this BBP. The effect of the external electric field on the $\Phi_{C T}$ electronic state accounts for finding the normalized direction, either, $\mathbf{l}_{1}$ or $\mathbf{l}_{2}$, which is privileged for the catalysis of this nucleophilic addition to an unsaturated electrophile group. In the present case both directions coincide with the reaction coordinate $\mathbf{C} \cdots \mathbf{O}$. This is the direction along which the electrons are reorganized to make the new bond, and this can either be assisted or inhibited by the external electric field. Paraphrasing Shaik, ${ }^{36,76}$ an external electric field oriented along the direction of the gradient vector of the BBP, that is $\mathbf{I}$ being the direction along which the bond changes occur, causes catalysis/inhibition of the reaction process by preferential lowering/raising the transition state and the reactant, depending if the relative direction of the field to the electronic flow that takes place through the l-direction.

An example of another type 1 corridor applied to catalysis is provided by the 1,2-sigmatropic $\mathrm{H}$ shift rearrangement of cyclopentadiene. This reaction was computationally studied by Schleyer ${ }^{77}$ and it was found that $\mathrm{Li}^{+}$was able to catalyze it. We take this reaction as an example of the present model. This chemical example is also proposed as a description of a primitive version of an algorithm based on Eq. (1) to find the optimal catalyst for a specific reaction. The first step consists to locate BBPs on the IRC path joining the reactants and products through the transition state. Since we are going from the reactant to the product we are only interested in the BBP located in the reactant valley. Note that according to the section Methodology this BBP usually is not exactly the optimal, however, it may be close to the optimal one. In the second step we compute and analyze the BBP gradient vector. In the next step we look for a chemical species that makes a force to the substrate in the direction to the BBP gradient vector. Note that in this situation, $d V_{\text {eff }}(\mathbf{r}) / d t \approx 0$, because, $|\mathbf{g}|-F \approx 0$, as explained in section 2. In our particular example the BBP structure and the corresponding gradient vector is shown in Figure 4, part (b). 
The selected chemical species which partially satisfies the above requirements is the $\mathrm{Li}^{+}$which was located below the molecular plane, that is to say in the opposite side where the H-shift takes place. According to the present theory the direction of the gradient vector at BBP in the presence of $\mathrm{Li}^{+}$should overlap as maximal as possible with the gradient vector of the BBP without the cation, otherwise, it is not the best catalyst. In the present case these two vectors form an angle of approximately $30^{\circ}$. We conclude that $\mathrm{Li}^{+}$is an efficient catalyst but is not the optimal. The structure of the BBP and the corresponding gradient vector is reported in Figure 4, part (b). The results and conclusions are in line with those reported in Ref. 77.

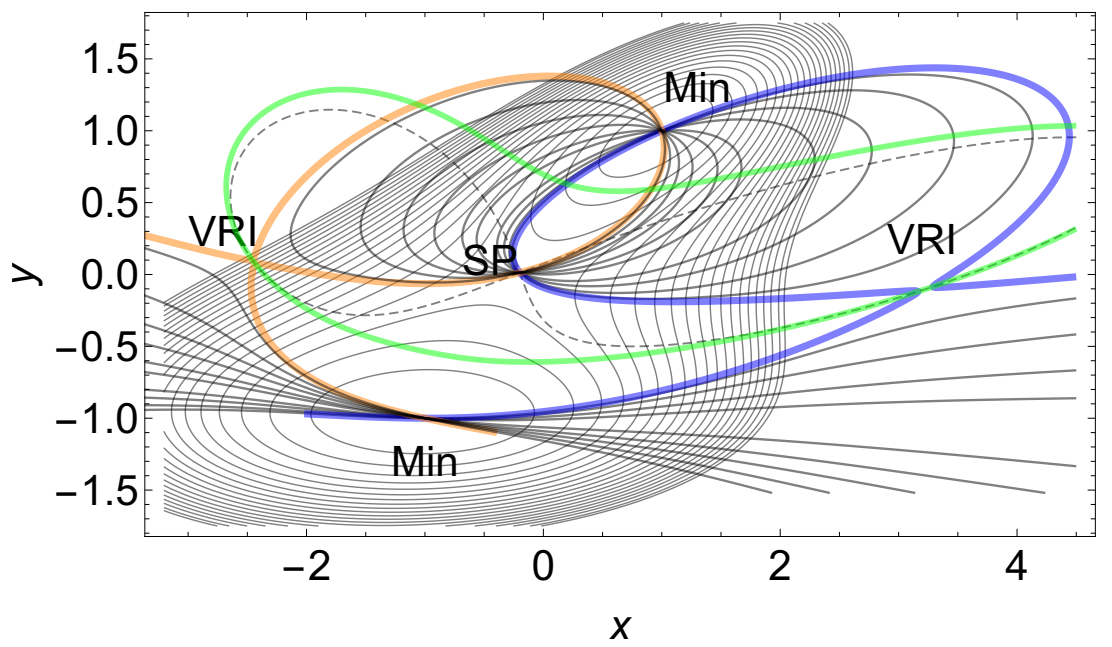

Figure 5: Roundabout half-corridor (above) of type 2 and useless half-corridors (below) of the PES of Eq.(S1) in SI. The NTs of the corridor fall apart into two branches. The set of circular branches characterize a chemical corridor for a pulling from the upper minimum to the lower one. The escaping NTs in the lower part indicate that a pulling in one of these directions is useless. None of the branches crosses either the BBP line (green) or the SP of index 1. These directions characterize a useless chemical corridor.

The PES displayed in Figure 1 also contains another type of corridor. In Figure 5 a useful halfcorridor for the upper minimum only is represented. It is filled with circular NTs which connect the upper minimum and the SP only and which cross the upper BBP line (the green line). This kind of corridor is named of type 2. Note that again the two singular NTs are the border lines of the corridor.

If we regard Figure 5 below, the half-family of NTs forms a contrast. Its branches start at the 
lower minimum at the point $(-1,-1)$. They cross neither the next BBP line nor the SP of index 1 . They characterize a forbidden chemical corridor for a pulling purpose. Note that in this case the pulling is not impossible but useless and leads in every case to an enforced reaction from the upper minimum to the lower one. We call this half-corridor a useless chemical corridor. As an example of this type of corridor, we could mention the failure of external forces to induce isomerizations of double bonds (i.e, E,Z transformations) reported in Refs. 78,79.

\section{Potential energy surfaces with alternate pathways}

Some chemical reactions take place through competing mechanisms. The topography of the PESs associated to these mechanisms consists in alternative pathways joining one reactant minimum to the same or different product minimums. Between the two SPs of index 1 can be an SP of index two. ${ }^{80-82}$ The difference between the alternative pathways is given by the barrier height of the corresponding SPs of index 1 and the possible existence or not-existence of minimums associated to intermediates. The existence or not-existence of intermediates can complicate the topological study of the pulling phenomena according to the basic Eq. (1). For this reason we present two cases in the following separated subsections. The examples analyzed and discussed below can be viewed and understood as topological maps from real chemical systems for pulling scenarios ${ }^{22}$ where the high dimension of the problem is projected into two intrinsic and distinguished dimensions. We use such a projection to draw chemical-physical consequences. We start with the simplest case of two parallel reaction pathways without an intermediate. ${ }^{83}$ This type of surface is relevant for modeling the so-called catch bonds. ${ }^{84-97}$

\subsection{Alternate elementary pathways: A modified Eckhardt-PES ${ }^{98}$}

We analyze the case of two competing reaction pathways without intermediates using a modified version of the Eckhardt PES ${ }^{98}$ (see Supplementary Information for its mathematical description and main features of this PES). The surface is constructed to realize that there is a slight asymmetry 


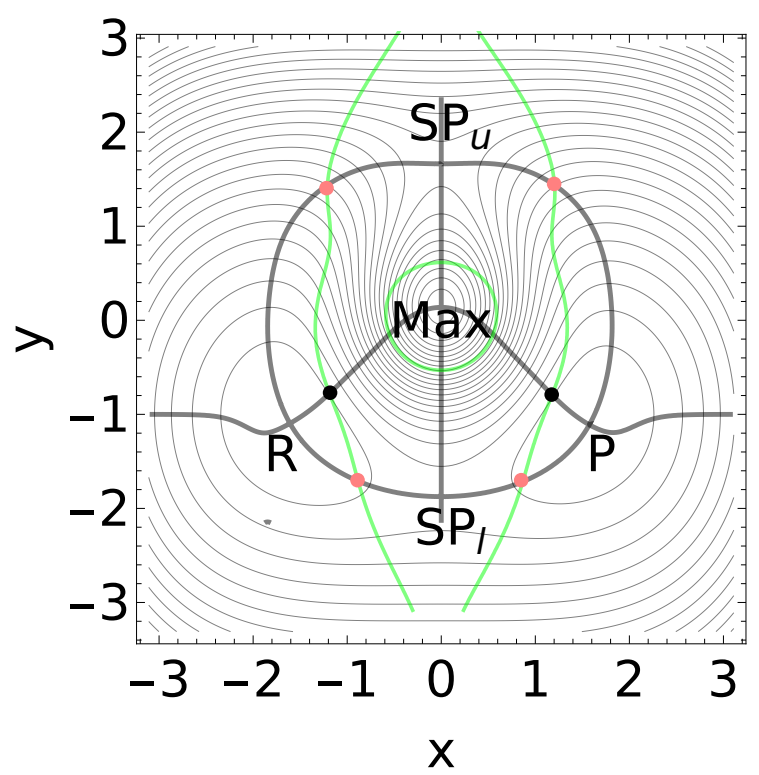

Figure 6: A modified Eckhardt surface ${ }^{98}$ with the stationary points, the minimums $\mathrm{R}$ and $\mathrm{P}, \mathrm{SP}_{l}$, $\mathrm{SP}_{u}$, and Max. The intersections of the GE curves (gray thick lines) with the BBP condition, $\operatorname{Det}(\mathbf{H})=0$, (green lines) give the optimal BBPs (red), or the VRI points (black). The optimal $\mathrm{BBP}_{1}$ is located at the point $(-0.89,-1.7)$, whereas the optimal $\mathrm{BBP}_{2}$ is at the point $(0.85,-1.7)$.

for the two minimums of the reactant, $\mathrm{R}$, and of the product, $\mathrm{P}$ and still more for the two SP of index 1, the low $\mathrm{SP}_{l}$ and the upper $\mathrm{SP}_{u}$. The PES has a heigh center, an SP of index two, labeled by Max, see Figure 6. As we can see with the level lines, on this PES, the upper reaction pathway taking place through the $\mathrm{SP}_{u}$ is energetically very higher with respect to the lower one that occurs through the $\mathrm{SP}_{l}$.

There are two VRI points between the reactant, R, and the maximum, as well as between the product, P, and the maximum, see Figure 6. Two singular NTs connect all these points shown in Figure 7. Further VRI points are outside the shown figure, near the $y$-axis through $x=0$.

Starting at the reactant, R, we find three families of NTs for a pulling to the two SP of index 1. One direct family is shown in Figure 8, and in Figure 9 we develop to one NT of the family the corresponding effective surfaces. Shown are three cases: panel (a) shows a low force, panel (b) a medium, and panel (c) shows a higher force. Figure 10 also explains a pathway to the lower $\mathrm{SP}_{l}$. Another way over the upper $\mathrm{SP}_{u}$ is treated by Figure 11. 


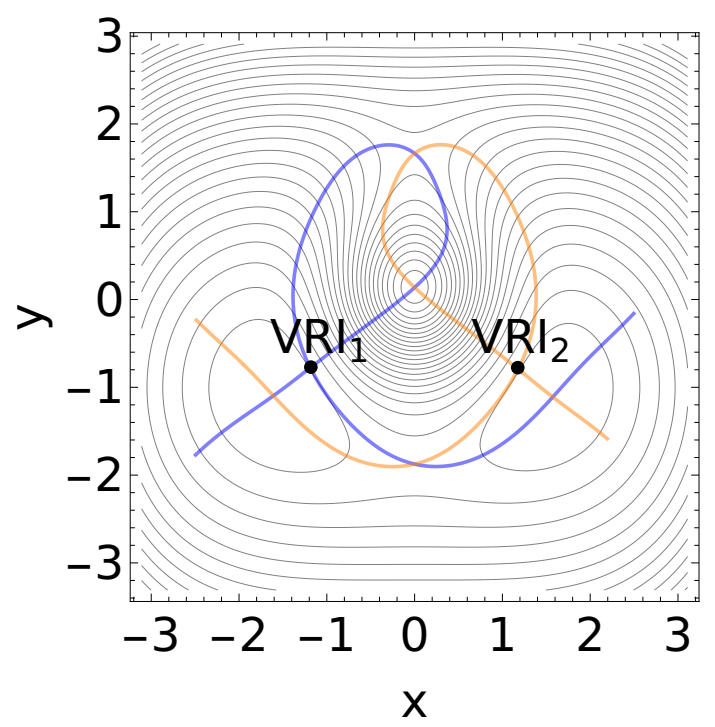

Figure 7: Singular NTs on the modified Eckhardt surface of Eq.(2) in SI. Each singular NT connects the respective VRI point with all stationary points of the surface. Blue: NT through $\mathrm{VRI}_{1}$, orange: NT through $\mathrm{VRI}_{2}$. Two branches of the respective singular NT form here a common loop.

The first family in Figure 8 realizes a direct connection between reactant, R, and product, P. This kind of direct chemical corridor is of type 1. In our two-dimensional problem is the corridor characterized by the set of all regular NTs located between the two singular NTs (which are depicted in blue and orange colors). The singular NTs are the borders of the corridor, compare the former Section. The chemical corridor contains the two optimal BBPs of the pulling, as well as of the reverse pulling, enforcing the optimal reactions from $\mathrm{R}$ to $\mathrm{P}$, or from $\mathrm{P}$ to $\mathrm{R}$. We recall that the optimal BBPs are at the intersection of a GE curve with the $\operatorname{Det}(\mathbf{H})=0$ or BBP manifold (green lines). These points are in the reactant or in the product valley. ${ }^{10}$ They are denoted by red points in the Figures. The realm of the corridor is a directional angle of $\approx \pi / 2$ for the gradient direction. It is here large because the reactant, $\mathrm{R}$, and the product, $\mathrm{P}$, are interconnected by a not strong curvilinear reaction coordinate. The respective optimal NT which meets the optimal BBP could be compared with a steepest descent curve from $\mathrm{SP}_{l}$, the Intrinsic Reaction Coordinate (IRC). ${ }^{62}$ However those parts of the NTs which come near to the VRI points are by no means a steepest descent curve. We need here with the class of NTs a new kind of curves to describe the pulling process. 


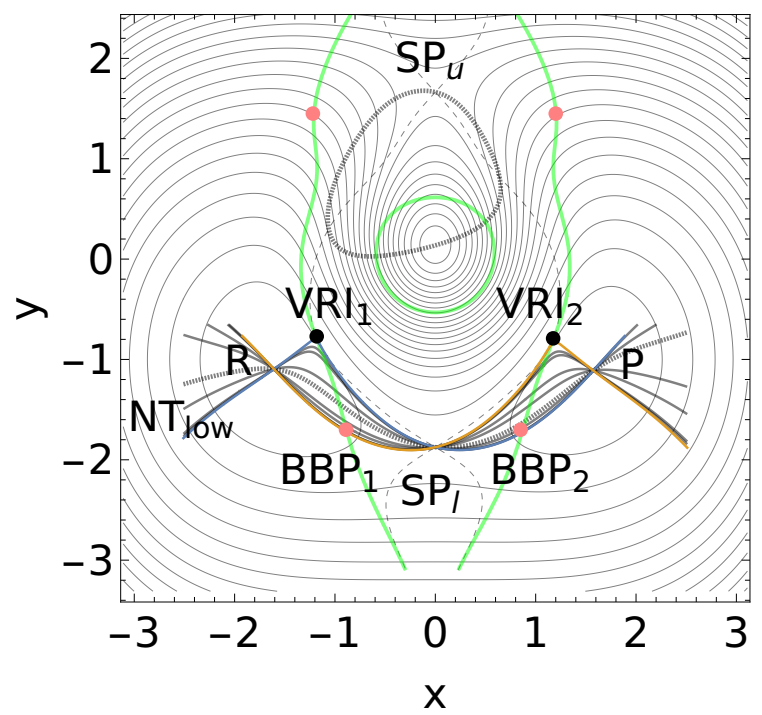

Figure 8: Direct pulling corridor of type 1 on the modified Eckhardt surface: all NTs going directly $\mathrm{R} \leftrightarrow \mathrm{P}$ form this family. Two singular NTs are the borders of the corridor which touch the VRIs, as well as the lower BBPs; they are colored by blue and orange. All NTs between the border lines belong to this corridor. The red points are the optimal BBPs. They are on the green curves which depict the condition $\operatorname{Det}(\mathbf{H})=0$. Small dishes depict the convexity border of the PES. The reaction valley region is in between the convexity border. A second, compact branch of all NTs goes through the $\mathrm{SP}_{u}$ and the maximum. The one loop of the gray, thick NT is shown alone. The surface without load is shown in the background for comparison.

The set of regular NTs that belongs to this type 1 chemical corridor satisfy the MEP condition ${ }^{73}$ since these NTs are located in the valley containing the minimums R and P and the SP of index 1. Pulling along any regular NT of this corridor leads to a BBP that is near to the optimal BBP, that is $\mathrm{BBP}_{1}$ if we are pulling from $\mathrm{R}, \mathrm{BBP}_{2}$ if we are pulling from $\mathrm{P}$. If we start a pulling at the $\mathrm{R}$ side, then the $\mathrm{SP}_{l}$ and the minimum $\mathrm{R}$ coalesce under this pulling, and the reaction is enhanced. We show in Figure 9 the action of a pulling along the thick gray, regular NT of Figure 8. It is named $\mathrm{NT}_{l o w}$. It is observed that the pulling force results in a displacement of the reactant and $\mathrm{SP}_{l}$ along this NT. As the magnitude of the force, $F$, increases, these two stationary points get closer and, eventually, they coalesce above a given external force (see Figure 9). The force direction is $\mathbf{l}=(0.983,0.185)^{T}$ and its $F_{\text {max }}=4.25$. The value of $F_{\text {max }}$ is the gradient norm at the intersection point between the regular NT and the BBP manifold depicted as a green line. In Figure 9 we shown three cases for $F$, namely, $F=1.4,2.8$ and 4.25 force in arbitrary units. For $F=4.25$, the 
lower $\mathrm{SP}_{l}$ and the reactant minimum, $\mathrm{R}$, coalesce at the $\mathrm{BBP}$ line, see panel (c). Indeed, the effective stationary points move on the same regular NT. The $\operatorname{Det}(\mathbf{H})=0$ condition also results for every $F$ in the same curve. For the regular NT of the pulling direction we show additionally its other compact branch passing through the $\mathrm{SP}_{u}$ and the maximum. On this branch the $\mathrm{SP}_{u}$ and the maximum move together. In this action the $\mathrm{SP}_{u}$ moves downhill in the direction to the reactant minimum $\mathrm{R}$, but the displacement is very small. For this reason the behavior of $\mathrm{SP}_{u}$ in this pulling action does not play an important role.
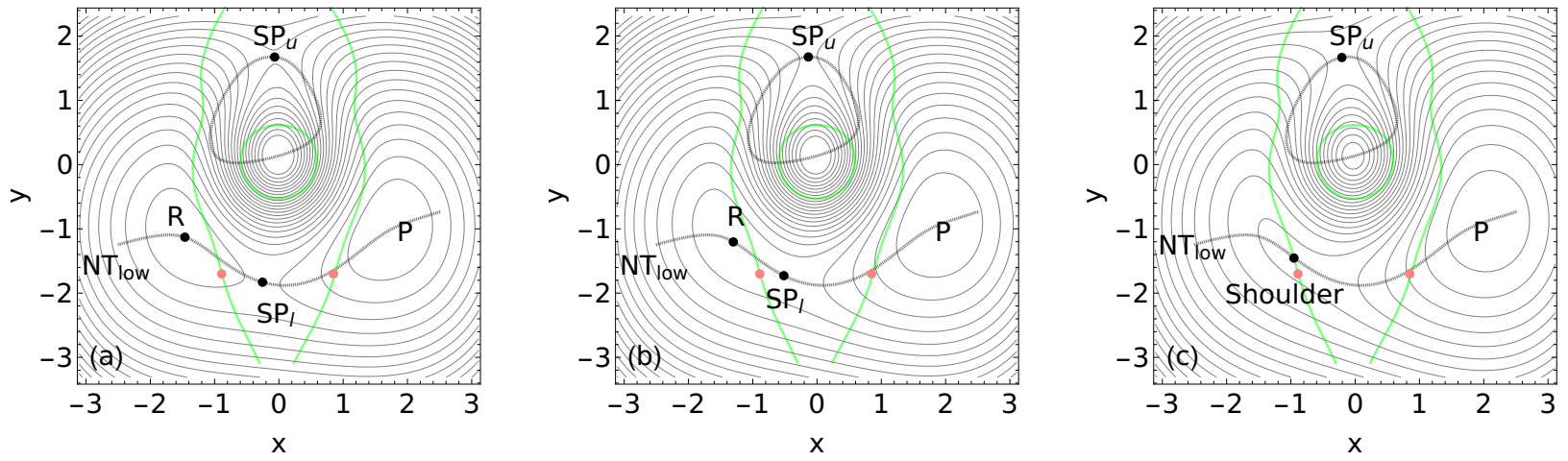

Figure 9: Effective PESs, $V_{f}$, of the modified Eckhardt surface ${ }^{98}$ with $\mathbf{l}=(0.983,0.185)^{T}$ and (a) $\mathrm{F}=1.4$, (b) $\mathrm{F}=2.8$, and (c) $\mathrm{F}=4.25$ arbitrary unit force. Here the localizations of $\mathrm{R}_{\text {eff }}, \mathrm{SP}_{l, e f f}$ and $\mathrm{SP}_{u, e f f}$ are marked by points.

Note that the force employed to induce the coalescence between reactant and $\mathrm{SP}_{l}$ in Figure 9 is larger than the optimal force, that is to say, the minimum force that is needed to favor the process. Interestingly, in this particular case, the optimal NT coincides with the singular NT depicted in orange in Figure 8, which defines the border of this corridor. The optimal $F$ value there is still smaller: $F_{\max }=2.956$. The direction of the optimal NT through $\mathrm{BBP}_{1}$ is $(0.878,-0.478)^{T}$. It is clear that the pulling is reversible by an inverse pulling direction. Under the inverse pulling, the lower $\mathrm{SP}_{l}$ and the product minimum move together and eventually coalesce. ${ }^{10,11}$ The VRI points, $\mathrm{VRI}_{1}$ and $\mathrm{VRI}_{2}$, are the border marks of the 'direct' family of NTs of type 1. The VRIs are the black bullets in Figure 6 to Figure 11 (excluding Figure 9). One should expect that such a described corridor is the usual case for pulling directions: in one direction, the reactant and an SP move together and form the well known transition state stabilization (TSS) of enzyme catalysis, ${ }^{46}$ 


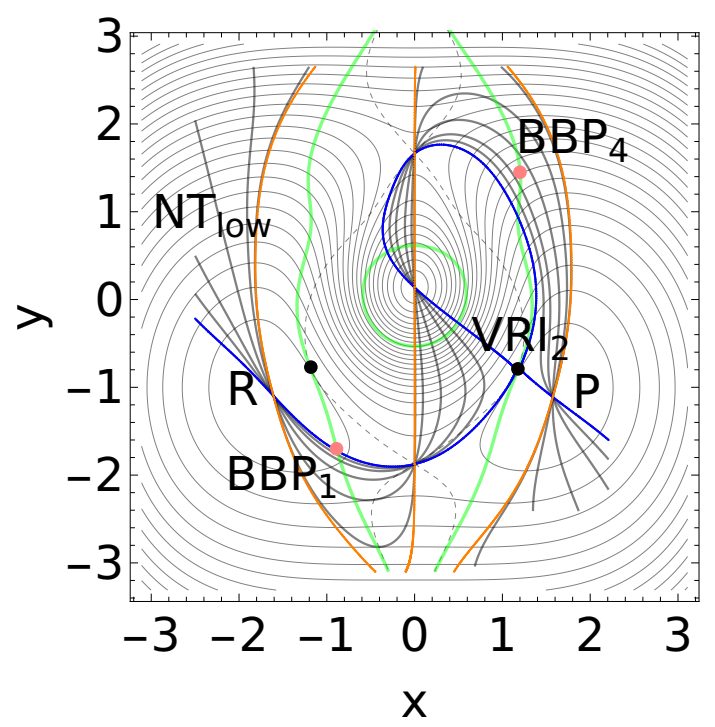

Figure 10: An asymmetric type 3 chemical corridor on the modified Eckhardt surface. ${ }^{98}$ All the NTs connect the minimums $\mathrm{R}$ and $\mathrm{P}$ in an indirect manner, i.e., they go through the $\mathrm{SP}_{l}$, Max, and $\mathrm{SP}_{u}$. Two singular NTs are the borders of this corridor; they are colored by blue and orange. The blue singular NT crosses the $\mathrm{VRI}_{2}$ two times in the product valley, the orange singular NT touches two VRIs outside of the Figure, near the $x=0$-line. The black points are VRIs. The red points are the optimal BBPs. The surface without load is shown in the background for comparison.

enhancing the reaction rate. But one should expect that pulling into the other, inverse direction makes that the product and this same SP move together and again form a TSS, now for the enhanced back-reaction. The reason for this expectation is simply the SP structure: in both directions of the SP valley the potential energy decreases along the steepest descent. However, we find below that the direct pulling corridor (of type 1) is usually very small, and that there other types exist.

Under the first corridor in Figure 8, the second family of NTs starts from minimum, R, to the lower $\mathrm{SP}_{l}$, see Figure 10 where again the border NTs are colored by blue and orange. However, its NTs turn after the lower SP uphill to the maximum in between the two SPs, then go to the upper SP, and further they turn back to the product, P. The possibility of a connection by regular NTs of an SP of index one and an SP of index two is given by the index theorem for NTs. ${ }^{99}$ Observe that the NTs of this corridor, especially the parts on the ridge between the $\mathrm{SP}_{l}$ and the $\mathrm{SP}_{u}$, are by no means any kind of minimum energy path. A further new property of the corridor is that it does not connect directly the reactant and the product. The effect of pulling on this family is very close to 


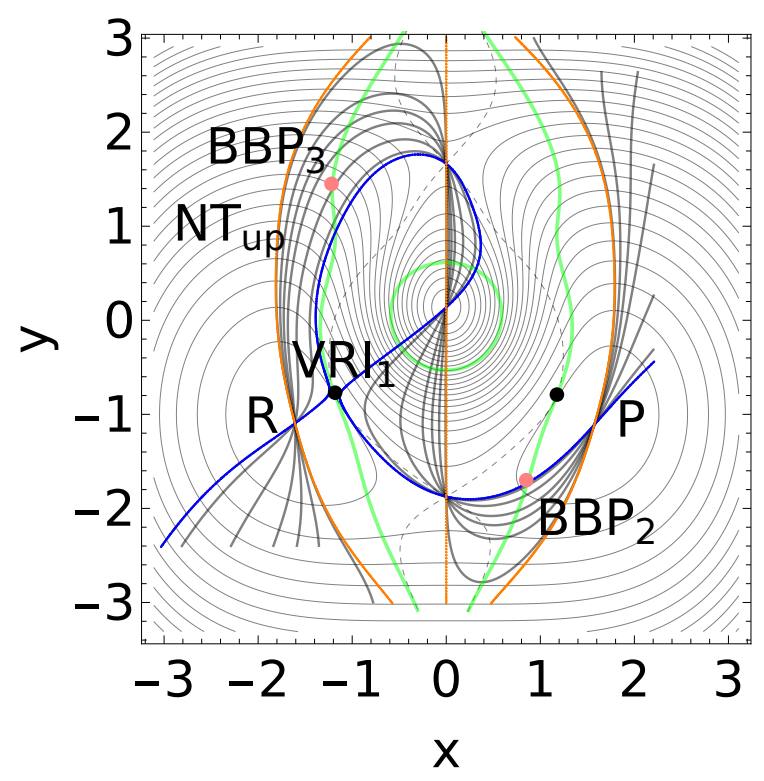

Figure 11: An asymmetric type 3 chemical corridor on the modified Eckhardt surface. ${ }^{98}$ All the NTs connect the minimums $\mathrm{R}$ and $\mathrm{P}$ in an indirect manner, i.e., they go through $\mathrm{SP}_{u}$, Max, and $\mathrm{SP}_{l}$. Two singular NTs are the borders of this corridor; they are colored by blue and orange. The blue NT touches the VRI 1 two times, the orange NT touches two VRIs outside of the figure, on the $x=0$-line. The red points are the optimal BBPs.

the previous studied case, namely, the reactant, $\mathrm{R}$, and the $\mathrm{SP}_{l}$ move together to form a TSS and coalesce at least. Now also the $\mathrm{SP}_{u}$ and the maximum move together. ${ }^{10,11}$ With this behavior, the family of NTs defines a new type of chemical corridor, labeled as chemical corridor of type 3 . Note that this corridor is indirect. In other words, this corridor does not go directly to the product. In this type, an inversion of the pulling direction starting from the second minimum, $\mathrm{P}$, does not lead to the $\mathrm{SP}_{l}$, it leads to the $\mathrm{SP}_{u}$. Thus the pulling along the type 3 corridor shows an intrinsic hysteresis. Of course, the hysteresis concerns here the mechanical corridor, its curve of FDSPs, but not possible chemical reactions over $\mathrm{SP}_{l}$. The reverse direction of pulling can turn the reaction of the molecule over the upper saddle. There the original SP height and the $F_{\max }$ at the respective $\mathrm{BBP}_{4}$ are much higher.

A representation of the effective PESs for different forces, $F$, for a pulling direction from a corridor of type 3 is given elsewhere. ${ }^{10,11}$ An important aspect emerging from the analysis presented above is that small changes in the direction of the external force in any chemical system statisfying an Eckhardt-like PES can result in drastically different scenarios due to the switch of 
the type of corridors. Starting from the minimum, $R$, only small variations of the pulling direction, l, can decide between a pulling effect of the type 1 or type 3 . The pulling itself works like in the first case: the $\mathrm{SP}_{l}$ and the reactant, $\mathrm{R}$, move together and coalesce, at least. However, at the same time, the $\mathrm{SP}_{u}$ and the maximum move together. ${ }^{10,11}$ Thus we find usually no TSS for the $\mathrm{SP}_{u}{ }^{11}$
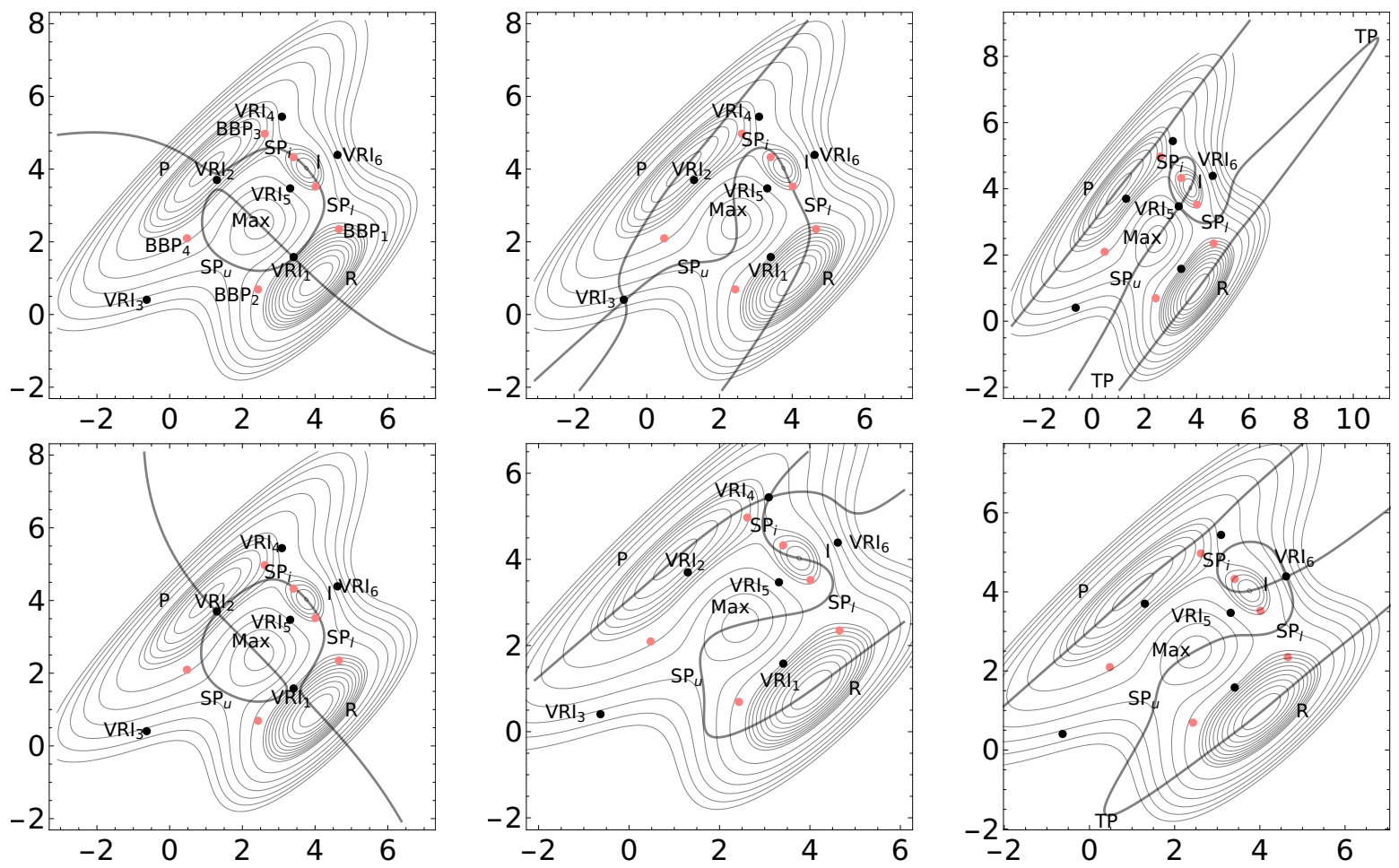

Figure 12: Singular NTs on the Rhee-Pande surface. (See Supplementary Information Eq.(S3).) The upper panels shows the NTs through the points $\mathrm{VRI}_{1}, \mathrm{VRI}_{3}$, and $\mathrm{VRI}_{5}$, but the lower panels shows the NTs through the points $\mathrm{VRI}_{2}, \mathrm{VRI}_{4}$, and $\mathrm{VRI}_{6}$. There are further VRI points outside of the region displayed, see Supplementary Information for further details about these VRIs.

Contrary to the lower $\mathrm{SP}_{l}$, to attain the higher $\mathrm{SP}_{u}$ from $\mathrm{R}$ by a pulling, we have to chose directions from a next family of NTs, shown in Figure 11. It is the mirror picture to the corridor of Figure 10. The mirror line is the $y$-axis for $x=0$. The optimal $\mathrm{BBP}_{3}$ of the family is at point $(-1.22,1.45)$. Its $F_{\max }=13.182$ is more than four times larger than the $F_{\max }$ at the lower $\mathrm{BBP}_{1}$. The direction of the optimal NT is $(0.502,0.865)^{T}$. After crossing the $\mathrm{SP}_{u}$, the optimal NT turns uphill and connects the two SPs, the $\mathrm{SP}_{u}$ and the $\mathrm{SP}_{l}$ and it still crosses the maximum, the SP of index two in between, before entering the product. If one pulls the reactant in any of the directions of 
this family, the upper SP and the reactant move together and coalesce, at least. However, on the other side of the PES, the lower SP and the maximum move together, thus, the barrier height of the lower SP increases under the pulling directions. It causes a decrease of the thermodynamical rate over this former lower SP, compare Ref. 11. This kind of an indirect corridor is again a pulling of type 3.

The deviation of pulling directions from a type 1, direct chemical corridor is expected to greatly increase for a high-dimensional PES. In two dimensions, like in our examples of the present study, there is one col direction along the SP and one orthogonal direction uphill the SP ridge. On an $N$-dimensional PES the one direction along the valley remains, however, there is an (N-1)dimensional ridge where orthogonal directions can break out off the col.

The three discussed corridors exhaust the full $360^{\circ}$ of pulling directions of the plane in the left minimum, R, in Figure 8, Figure 10 and Figure 11, as well as in the right minimum, P. Thus, here "forbidden" 11 or useless directions do not exist. The case is newly discussed in the next subsection.

The topography of the Eckhardt surface might be relevant, for instance, to rationalize the chemistry associated with the cis $\leftrightarrow$ trans isomerization of azobenzene derivatives, which constitute one of the most important classes of photoswitchable molecules. It has been observed that this isomerization can take place by means of two different reaction pathways: a rotation mechanism and an inversion mechanism. ${ }^{100}$ Remarkably, pronounced hysteresis was detected in simulations in which the isomerization was induced mechanically (either with a pull or push force). ${ }^{101}$

The ring opening in benzocyclobutene $(\mathrm{BCB})$ is a next chemical example. ${ }^{31} \mathrm{BCB}$ acts as a twofold proof of our concept.

(i) An appropriate pulling force can enforce a symmetry forbidden reaction path, a conrotatory ring opening against the Woodward-Hoffmann rules. Thus, the respective pulling drives the molecule over a higher SP of the PES.

(ii) By the use of two experimentally different pulling directions one gets two different amounts of force or a literally opening of the molecule, $F_{1}=1400 \mathrm{pN}$ against $F_{2}=900 \mathrm{pN}$. We assume that 
both directions are in the same corridor. However, the height of their BBP energies can be very different. ${ }^{11}$ Direction $\mathbf{l}_{1}$ is the pulling mainly along a single bond, $\mathbf{l}_{2}$ is the pulling mainly along a double bond. The forces are applied by a direct anchor or a so-called lever-arm polymer anchor of the molecule. ${ }^{31}$

\subsection{Alternate Pathways with Intermediates: The Rhee-Pande PES ${ }^{102}$}

The examples studied in the previous subsection concern alternate pathways without intermediates. In this subsection we study the case of alternate pathways with an intermediate and its possible effect on the pulling. We consider the case of the Rhee-Pande PES. ${ }^{102}$ As before the surface has two alternate pathways ${ }^{86,93}$ where we can assume that different cases of a directed force, $\mathbf{f}$, again enable two different pulling pathway families with respective FDSPs. One sort of corridors leads over the low energy SP, but the other SP is energetically disfavored in the forceless case. ${ }^{59}$ Of course, the two-pathway-model is still in the framework of minimal models, ${ }^{55}$ however, it easily illustrates the theory outlined in section 2. For the two-dimensional test surface of Rhee and Pande ${ }^{102}$ for the case of competing RPs an intermediate minimum emerges on the lower path. The intermediate is behind the lower SP and it has no influence on possible reaction rates for $\mathrm{R} \rightarrow \mathrm{P}$ reactions on this PES, ${ }^{11}$ however, here we will observe new types of pulling corridors. Again, between the two competing SPs emerges a maximum, an SP of index two. The mathematical expression and the main features of the Rhee-Pandee PES are detailed in the Supplementary Information.

The putative simplicity of this two-dimensional PES model should not conceal our view on the variety of pulling corridors. To study the possible pulling corridors, we first show the singular NTs which form the corresponding borders of the corridors, in Figure 12. In comparison to the former example, there emerge the additional $\mathrm{VRI}_{3}$ and $\mathrm{VRI}_{4}$ in the product valley. Because there an intermediate minimum exists, we find further VRI points on the central ridge of the surface, the $\mathrm{VRI}_{5}$ and $\mathrm{VRI}_{6}$, around the intermediate. A further $\mathrm{VRI}_{7}$ is discussed below; and we found two extra VRIs that lie far outside of the region of interest from the chemical point of view. Note that two branches of the singular NTs through $\mathrm{VRI}_{1}, \mathrm{VRI}_{2}, \mathrm{VRI}_{5}$ or $\mathrm{VRI}_{6}$ form loops. However, the 
singular NTs through the $\mathrm{VRI}_{3}$ or $\mathrm{VRI}_{4}$ are globally correct pitchfork bifurcations. ${ }^{69}$

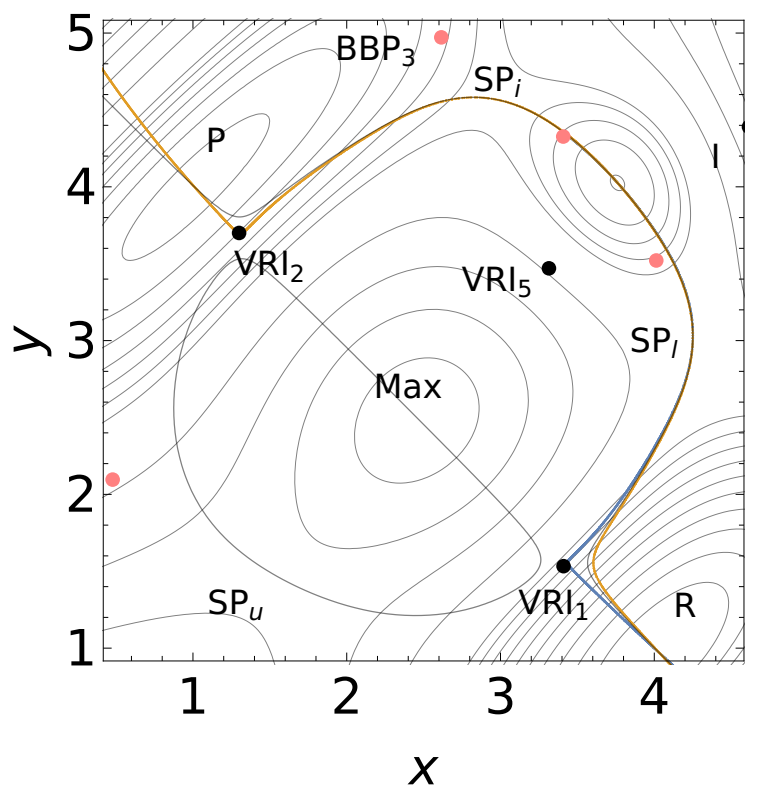

Figure 13: The direct small corridor between the two deep minimums, $\mathrm{R}$ and $\mathrm{P}$. The border parts of the two singular NTs are drawn in blue (the NT throughVRI ${ }_{1}$ ) and orange color (the NT through $\mathrm{VRI}_{2}$ ). A usual regular NT is drawn by a gray line in between. It has a second branch through Max and $\mathrm{SP}_{u}$.

There is a direct corridor named pulling of type 1, shown in Figure 13, like in the former examples. This particular corridor is circumscribed by two singular NTs that mark the limits of a very small region of the configuration space. It is quenched between the two singular NTs through $\mathrm{VRI}_{1}$ and $\mathrm{VRI}_{2}$, compare the left two panels of Figure 12. An NT inside the corridor is also shown in gray color; it has a second, closed branch through the maximum, and near the two VRIs, and through the $\mathrm{SP}_{u}$. Of course, this second branch concerns the movement of the $\mathrm{SP}_{u}$ and of the maximum under a pulling along the direction of the included NTs of the type 1 corridor. The $\mathrm{SP}_{u}$ also moves downhill in direction of the minimums, R or P, along the NT. The movement however is smaller than that of the minimums, $\mathrm{R}$ or $\mathrm{P}$, and the $\mathrm{SP}_{l}$ together. The small changes in $\mathrm{SP}_{u}$ thus do not have relevant consequences from a chemical point of view when a force is applied to the corridor going through $\mathrm{SP}_{l}$. The corridor itself concerns the pulling over the low $\mathrm{SP}_{l}$. 

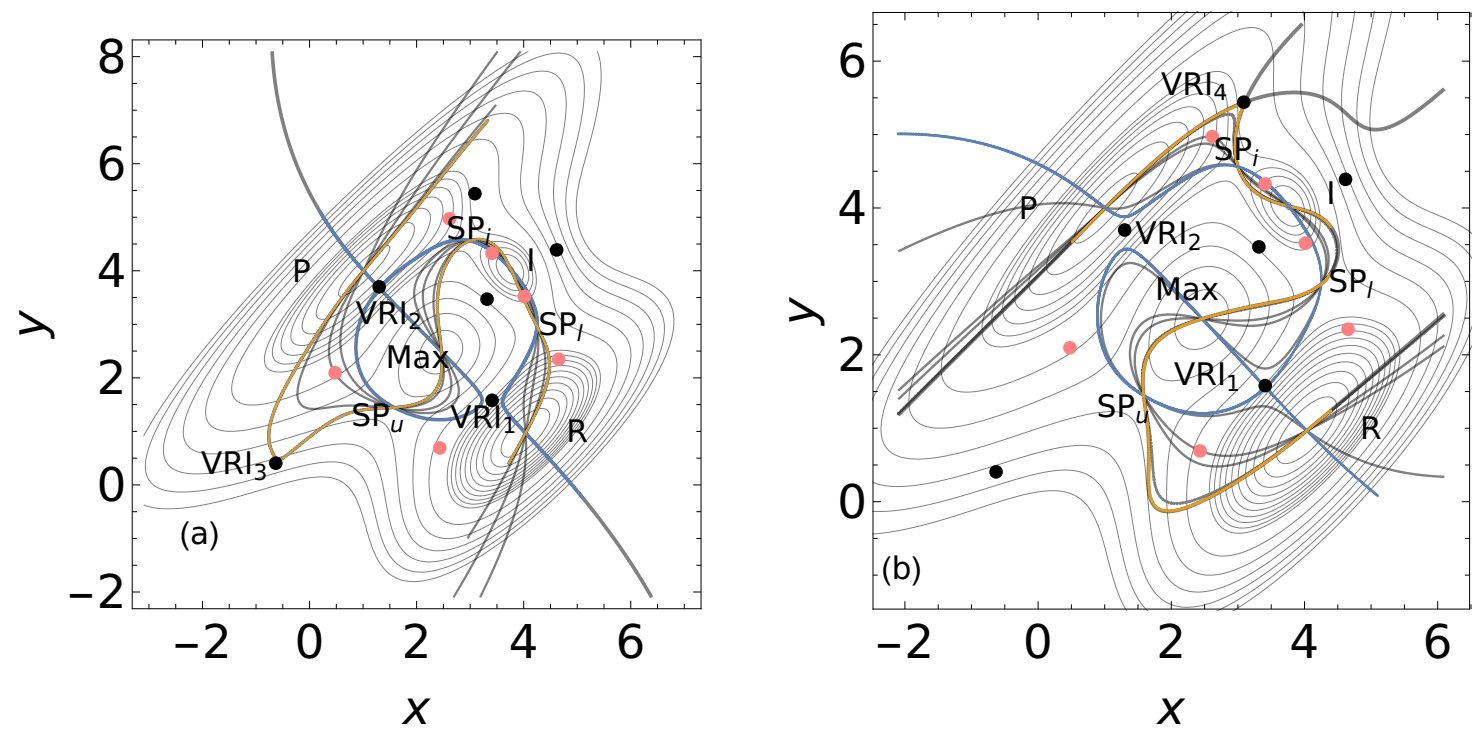

Figure 14: Corridors of type 3. (a) Corridor between $\mathrm{VRI}_{2}$ and $\mathrm{VRI}_{3}$, but also $\mathrm{VRI}_{1}$ is almost thouched. Some normal NTs are drawn by gray lines, but the border parts of the two singular NTs are drawn in blue and orange color. Optimal BBPs are red points. (b) Corridor between $\mathrm{VRI}_{1}$ and $\mathrm{VRI}_{4}$, here $\mathrm{VRI}_{2}$ is almost thouched. The surface without load is shown in the background for comparison.

Two further corridors are indirect connections by NTs of the reactant, R, and the product, P. They are formed similar to the former case of the modified Eckhardt surface. They are indirect corridors of pulling type 3 . One case is the corridor between the $\mathrm{VRI}_{2}$ and $\mathrm{VRI}_{3}$, see Figure 14, panel (a) and the other case is the corridor between the $\mathrm{VRI}_{1}$ and $\mathrm{VRI}_{4}$ in panel (b).

The type of corridors displayed in Figure 15 and Figure 16 show a complicated behavior but follow the features of the precedent corridors. These corridors include useless half-corridors like that found in the description of the corridor of type 2. However, they are more complicated due to the higher complexity of the underlying PES. Nevertheless, starting at reactant, R, and using such a corridor enforces the reaction over $\mathrm{SP}_{l}$ in case of Figure 15, or over $\mathrm{SP}_{u}$ in case of Figure 16.

In these corridors the former relation between reactant, $\mathrm{R}$, and the product, $\mathrm{P}$, is fully disconnected. This kind of corridors is named pulling of type 4. They start in the reactant region, at $\mathrm{R}$ , but after crossing the region of the maximum, they do not turn down to the product, however, they escape uphill into the mountains of the surface. The first case is the corridor between $\mathrm{VRI}_{3}$ 

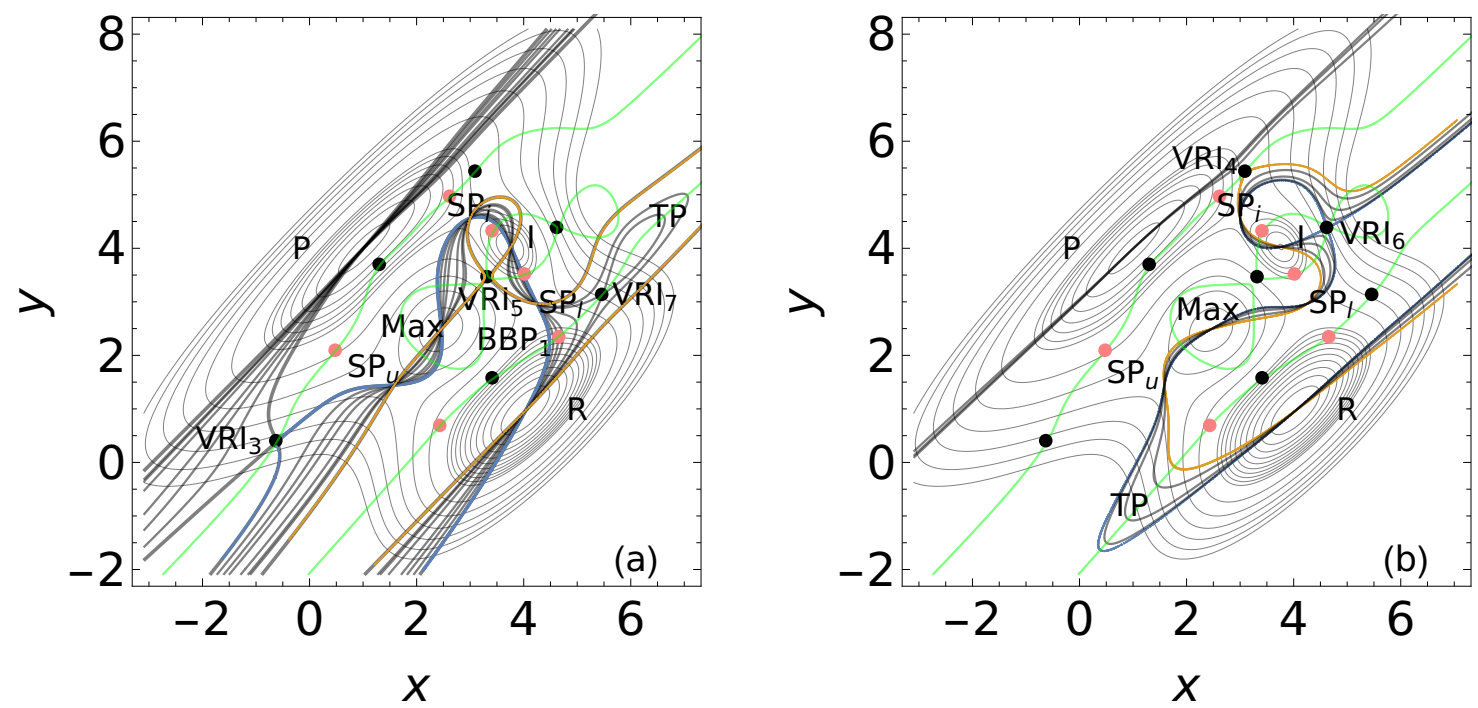

Figure 15: Corridors of type 4. (a) The corridor between the $\mathrm{VRI}_{3}$ and $\mathrm{VRI}_{5}$ is very 'unsymmetric'. The singular border parts are drawn in blue and orange color. The corridor does not reach the product, however, escapes uphill after $\mathrm{SP}_{u}$. Note that some NTs of this corridor have turning points (TP). (b) The corridor between the $\mathrm{VRI}_{4}$ and $\mathrm{VRI}_{6}$ is also very 'unsymmetric'. It escapes uphill after $\mathrm{VRI}_{6}$. All NTs of this corridor have TPs between the start in $\mathrm{R}$, and the $\mathrm{SP}_{u}$. The surface without load is shown in the background for comparison.

and $\mathrm{VRI}_{5}$, see Figure 15 (a). It goes further uphill after $\mathrm{SP}_{u}$. At the beginning of this corridor, a further peculiarity emerges by a $\mathrm{VRI}_{7}$, compare Figure 15. This VRI point is not a border point of a corridor. The two upper branches of the singular NT form a compact loop like the NTs through the $\mathrm{VRI}_{1}, \mathrm{VRI}_{2}, \mathrm{VRI}_{5}$ and $\mathrm{VRI}_{6}$. Here, the loop does not cross further stationary points of the surface. ${ }^{103}$ The normal NTs of the corridor can circumvent the singular point, and also the loops in the back of it.

The closed circular branches of NTs which form the loop 'behind' $\mathrm{VRI}_{7}$ form a region of the PES which is not connected to the minimum, R, by a continuous NT. Such compact branches of NTs are described elsewhere. ${ }^{103}$ The unreachability of special regions, seen from the minimums, also concerns the regions 'behind' the $\mathrm{VRI}_{3}$ and the $\mathrm{VRI}_{4}$.

The other corridor in the right panel of Figure 15 is the small corridor between $\mathrm{VRI}_{4}$ and $\mathrm{VRI}_{6}$. It goes further uphill after $\mathrm{VRI}_{6}$.

If one looks to the product side, there is a disconnected second half-corridor in the product 
valley, in both cases. (Disconnected with the exception of the one singular NT forming the border: at the corresponding singular VRI point, the two corridors touch.) The regular NTs of the product side do not cross the (green) BBP line. It means that an inverse pulling is quite useless: it moves the product minimum in its valley, forward or backward, but not to a coalescence with one of the SPs. A pulling in such a direction cannot enforce the disappearance of the product side, independent from the amount of the force, $F$.

The last corridor is a circular region shown in Figure 16. It starts at R, uphill to a TP, then downhill to one of the two SPs, $\mathrm{SP}_{u}$ or $\mathrm{SP}_{l}$, further to the maximum in the central region, and over the contrary SP, and a corresponding TP back to the reactant minimum. The disconnection between the reactant, $\mathrm{R}$, and the product, $\mathrm{P}$, is still stronger realized. This kind of a roundabout corridor is again a pulling of type 2 . The orange singular NT has a turning point (TP) near $(0,-2)$. There the minimum, $\mathrm{R}$, and the upper $\mathrm{SP}_{u}$ would coalesce if the force points in direction of this NT, and the amount, $F$, is high enough. For the contrary case of an inverse force, the corresponding TP of the blue singular NT is near point $(10,8)$. There the minimum, $\mathrm{R}$, and the lower $\mathrm{SP}_{l}$ would coalesce. Also the intermediate and the $\mathrm{SP}_{i}$ then coalesce on one of the circles left and above the VRI points. For a pulling in this roundabout corridor we find a simple switch between two competing SPs. They are enforced by the same force, only in forward or backward direction. Of course, the BBPs of special NTs can be of quite different height.

If one looks to the product side, there is a truly disconnected half-corridor in the product valley. The NTs of the product side do not cross the (green) BBP line. It means that an inverse pulling is useless from point of view of the product side. It moves the product minimum in its valley, for or back, but not to a coalescence with one of the SPs. A pulling cannot enforce a disappearance of the product side, independent from the amount of the force, $F$. The type 2 pulling corridor is in every case of a corresponding force a 'one-way' enforced reaction $\mathrm{R} \rightarrow \mathrm{P}$. In the forward direction, say with $F>0$, it may lead over $\mathrm{SP}_{l}$. In the backward direction, say with $F<0$, it may lead over $\mathrm{SP}_{u}$. No $F$ can enforce the back-reaction, $\mathrm{P} \rightarrow \mathrm{R}$. 


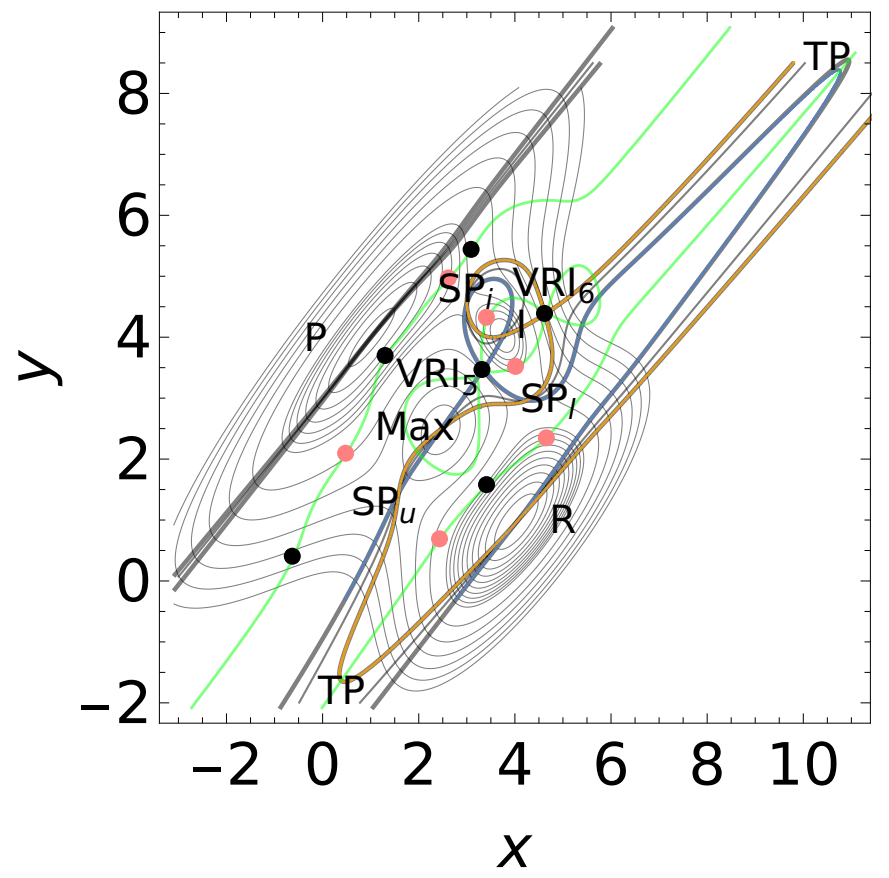

Figure 16: The circular corridor between the border points $\mathrm{VRI}_{5}$ and $\mathrm{VRI}_{6}$. A regular NT is drawn by a gray line in between the borders. The border parts of the two singular NTs are drawn in blue and orange color.

There are no further directions for a pulling or pushing of the molecule at its reactant state. The six given corridors exhaust the full $360^{\circ}$ directions of the right minimum. Only the forward $(F>0)$ or backward directions $(F<0)$ describe a useful pulling, or a useless one. Thus, "forbidden", or useless directions do not exist for the reactant, ${ }^{11}$ but thoroughly for the product side, compare the Figure 15 and Figure 16.

We emphasize that for directions of all types of corridors we can here enforce the reaction $\mathrm{R} \rightarrow \mathrm{P}$. All pullings make that the reactant, $\mathrm{R}$, and a respective $\mathrm{SP}$ move together, along the respective NT, and coalesce at least, if $F_{\max }$ at the $B B P$ is fulfilled. The different types of corridors concern, for this PES model, a possible question for a back-reaction only.

A key chemical process whose mechanism can be explained through a Rhee-Pande-like surface is the Diels-Alder reaction under the influence of an external electric field. ${ }^{34,76}$ The electric field applies directly through Eq. (1) on the PES of the system. ${ }^{29}$ When a negative electric field is 
oriented along the reaction axis, the reaction takes place via a two-step mechanism with a zwitterionic intermediate if and only if the electric field is larger (in absolute value) than a given threshold. ${ }^{76}$ According to the present theory, this threshold is associated with the force of the optimal BBP point. On the contrary, for smaller fields or fields pointing in the opposite direction, the reaction takes place via a concerted mechanism. ${ }^{76}$ Therefore, fields in opposite direction pull over different SPs, which gives rise to hysteresis. Different directions, $\mathbf{l}_{i}$, of the force are automatically given by different enantiomeric forms of the used molecule. ${ }^{34}$ In a well adapted direction, the force of the field causes a fivefold increase of the reaction rate. On the other hand, the electric field can be turned back. A reversed field makes an increase of the $\Delta E^{\ddagger}$ for an effective TS. Thus, the reversion of the field does not lead to a TSS in the other direction, for the back-reaction. It could be a hint to the chemical corridor of type 3 where the NTs turn up after the SP to a maximum of index two on the PES, compare the Figure 10,

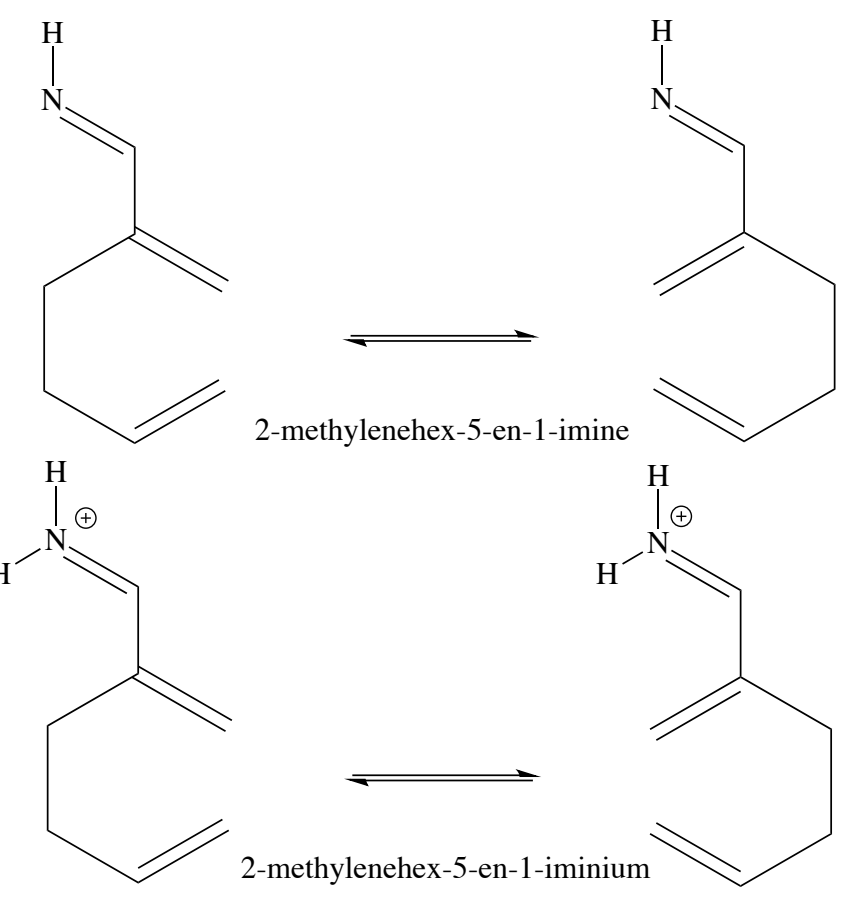

Figure 17: Cope reaction of 2-methylenehex-5-en-1-imine and the 2-methylenehex-5-en-1iminium ion.

An example that shows the application of the present theory to a catalytic process based on 
the Rhee-Pande model is the Cope rearrangement, ${ }^{104}$ see Figure 17. The Cope reaction stands as an example of [3,3]-sigmatropic rearrangement. It involves a stereospecific and concerted migration of an allyl group along a second allyl fragment within a 1,5-diene with concomitant $\sigma$ - and $\pi$-bond reorganization. ${ }^{105}$ This reaction is mainly reversible and very often needs strong reaction conditions. For the reason it is important to develop catalytic variants of the Cope rearrangement. Such developments are the metal-catalysts by Pd(II) ${ }^{106}$ and gold by bonding to the diene $\pi$-system and the substituents attached to the 1,5-diene. In the latest case the catalytic rate process can be enhanced if the substituents are electron-withdrawing with the addition of Brønsted acids. ${ }^{107}$ Examples of these substituents are carbonyls and the iminium ions recently reported by Gleason and Kaldre. ${ }^{108,109}$ We proof by doing the present theory that electron-withdrawing groups transformed in ions enhance its catalytic rate. For the purpose we compute at HF/6-31G level of theory the Cope rearrangement of 2-methylenehex-5-en-1-imine, see Figure 17, both, the concerted and the non-concerted pathways. The non-concerted path is the lowest energy path, passing through a biradical intermediate, see Figure 18 (a).

The two transitions states joining this intermediate with the reactant and product minimums have a biradical character. The transition state which corresponds to the concerted path does not show biradical character. From this description and regarding the energy profile we can conclude that the PES of the Cope rearrangement of the 2-methylenehex-5-en-1-imine is topographically analog to the Rhee-Pande surface analyzed above. The BBPs were obtained from the calculation of the IRC curves for the concerted and the non-concerted pathways. These BBPs are approximated; they are not necessarily the optimal ones. They might not belong to a gradient extremal curve. The structures of the six BBPs are given in Figure 19 with the corresponding components of their gradient vector. According to the present theory, the analysis of these gradient vectors gives us the optimal external force to be applied to enhance the rate of the process. According to that explained in references 108,109 the protonated form enhances the rate of reaction. We proof that this experimental observation is in accord to the present theory. First we add a proton to each BBP structure and compute the gradient. The protonated BBP structures are the corresponding BBPs 
a)
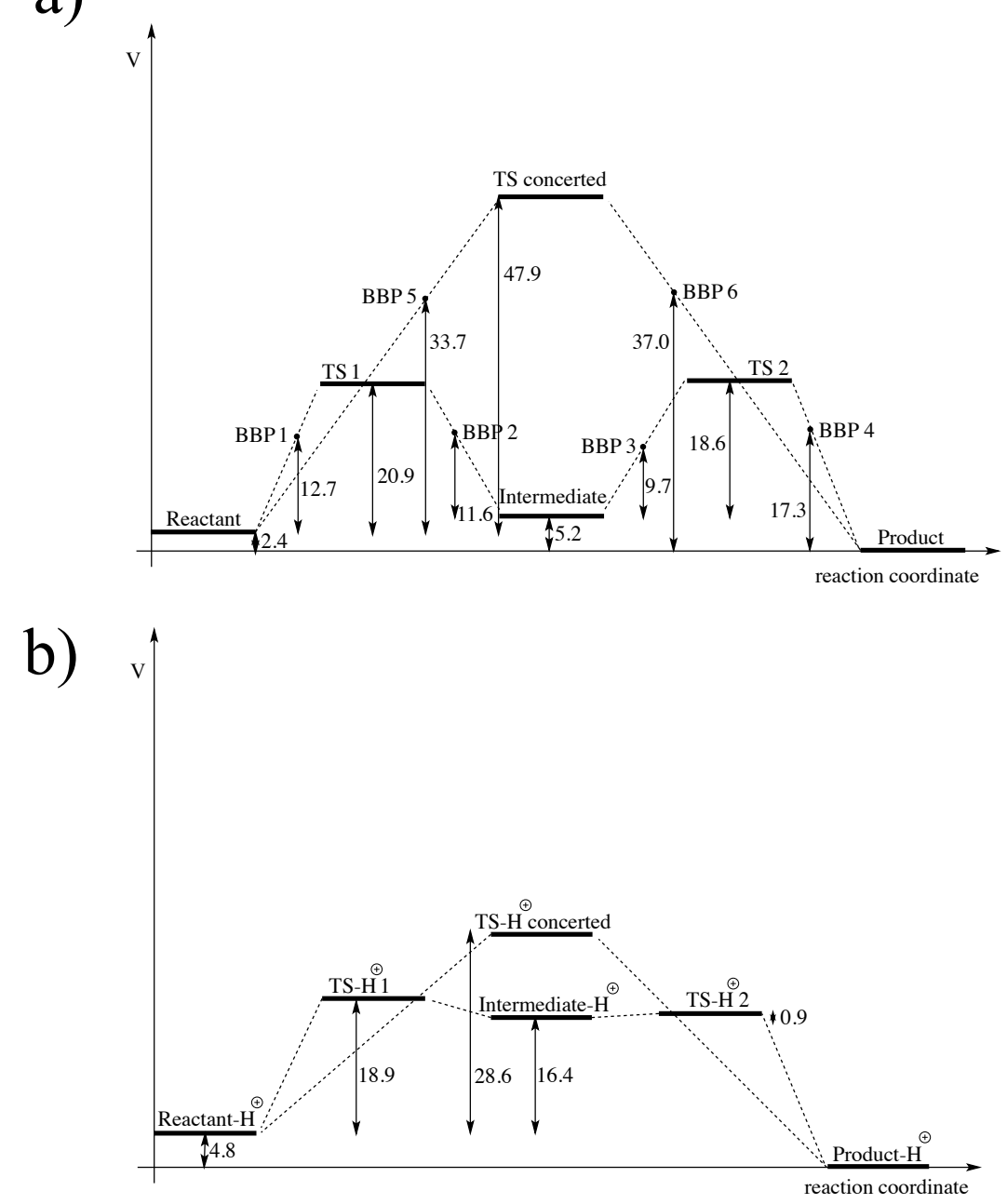

Figure 18: Energy profiles of the Cope rearrangement of the (a) 2-methylenehex-5-en-1-imine, (b) 2-methylenehex-5-en-1-iminium ion. The energies are given in $\mathrm{kcal} \mathrm{mol}^{-1}$.

of the 2-methylenehex-5-en-1-iminium ion, see Figure 17. If we compare the normalized gradient vector under exclusion of the proton component with the normalized gradient vector of the original BBP structure, we observe a very good concordance.

More specifically, the new gradient on the original BBP geometry provoked by the presence of the proton, $\mathbf{g}$ ' $=\mathbf{l}^{\prime}\left|\mathbf{g}^{\prime}\right|$, where the gradient vector $\mathbf{g}$ ' and the normalized vector $\mathbf{l}^{\prime}$ do not have the proton components, forms with the original BBP gradient vector, $\mathbf{g}=\mathbf{l}|\mathbf{g}|$ an angle near to $60^{\circ}$. With this result, finally we compute the reaction pathways of 2-methylenehex-5-en-1-iminium ion 


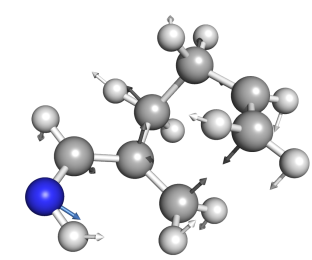

BBP 1

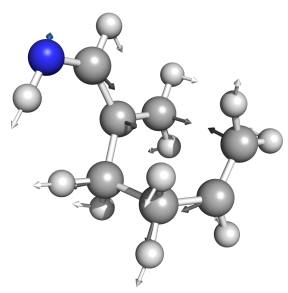

BBP 4

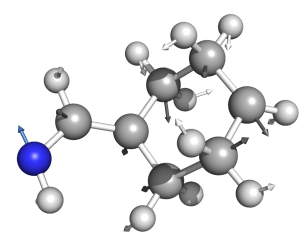

BBP 2

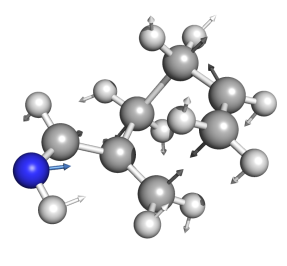

BBP 5

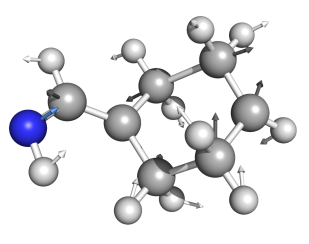

BBP 3

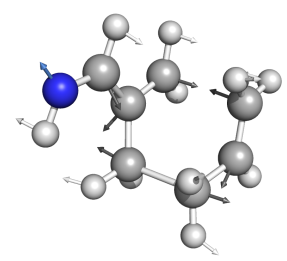

BBP 6

Figure 19: Structures of the BBPs for the concerted and non-concerted pathways corresponding to the 2-methylenehex-5-en-1-imine Cope rearrangement. The labels are that given in Figure 18 (a). The arrows are the gradient components.

at the same level of theory. The energy profiles for the concerted and non-concerted pathways are reported in Figure 18 panel (b). The form of this profile is in accordance to the behavior described in Figure 14 panel (a) of the Rhee-Pande surface. From these results we conclude that the protonation is an efficient catalyst however it is not the optimal catalyst according to the present theory.

\subsection{Alternate pathways with different products: a modified BQC PES ${ }^{110}$}

In this subsection we deal with the case of alternative pathways, each of them leading to a different product. Additionally, the products can be connected through another pathway. Such PESs are related with mechanisms of reactions where the reactant converts to different products.

The particular PES employed in this subsection, which is a modified version of a surface proposed by us, ${ }^{110}$ is shown in Figure 20 (the mathematical expression A reaction path opens from 


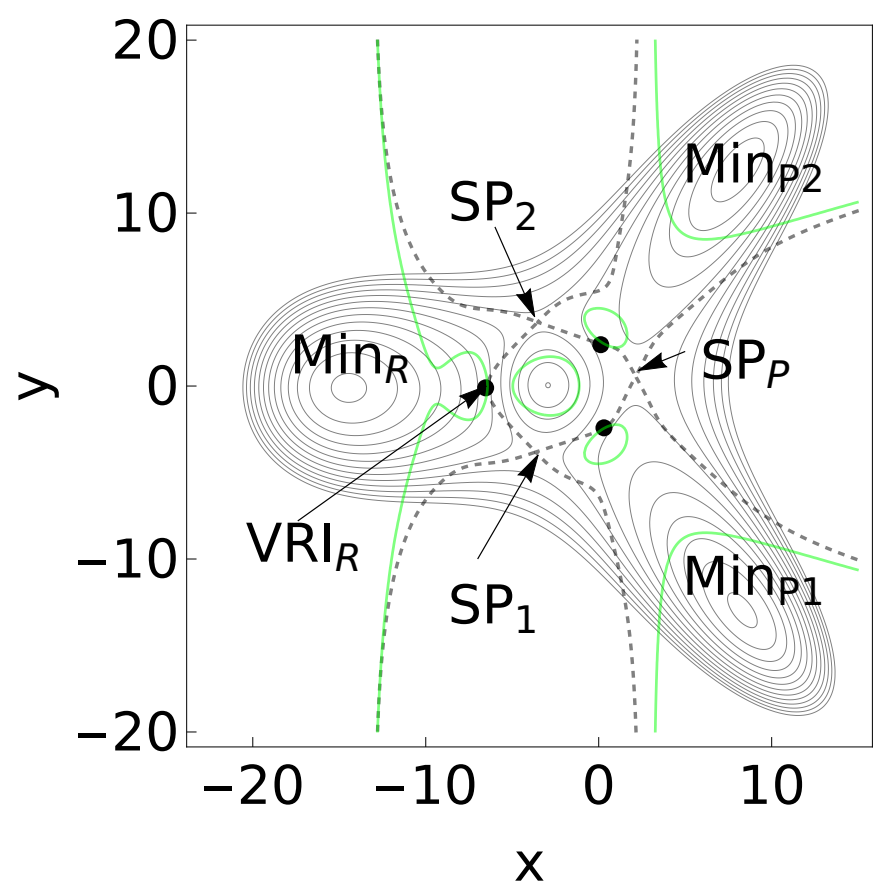

Figure 20: A modified BQC surface with the stationary points and some VRIs (black bullets), see E.(4) in SI. The green lines correspond to the BBP condition $\operatorname{Det}(\mathbf{H})=0$. The dashed line is the convexity border of the PES.

$\mathrm{R}$ to $\mathrm{P}_{1}$ right below, and a second reaction path opens from $\mathrm{R}$ to $\mathrm{P}_{2}$ right top, and also a valley exists between $\mathrm{P}_{1}$ and $\mathrm{P}_{2}$. In sum we have a curvilinear triangle of reaction pathways. The SPs are separated by VRI points. The center is again an SP of index two. There is a slight asymmetry for the two SPs: the $\mathrm{SP}_{1}$ is slightly lower in energy than the $\mathrm{SP}_{2}$.

In contrast to the first modified Eckhardt case, here we find three direct corridors of type 1, along the three assumed reaction pathways. To get the corridors, we have to set first the borders, the singular NTs of this PES. They are shown in Figure 21. There are valley-ridge inflection points between the reactant and the maximum, as well as between the two product minimums and the maximum, see Figure 21. Panel (a) shows the singular NT through $\mathrm{VRI}_{1}$, panel (b) the singular NT through $\mathrm{VRI}_{R}$, and panel (c) shows the singular NT through $\mathrm{VRI}_{2}$. Further VRI points are discussed later.

The singular NTs are the borders of the possible pulling corridors on the BQC surface. The three direct corridors are obtained for NTs between the singular NTs. Two direct corridors are 

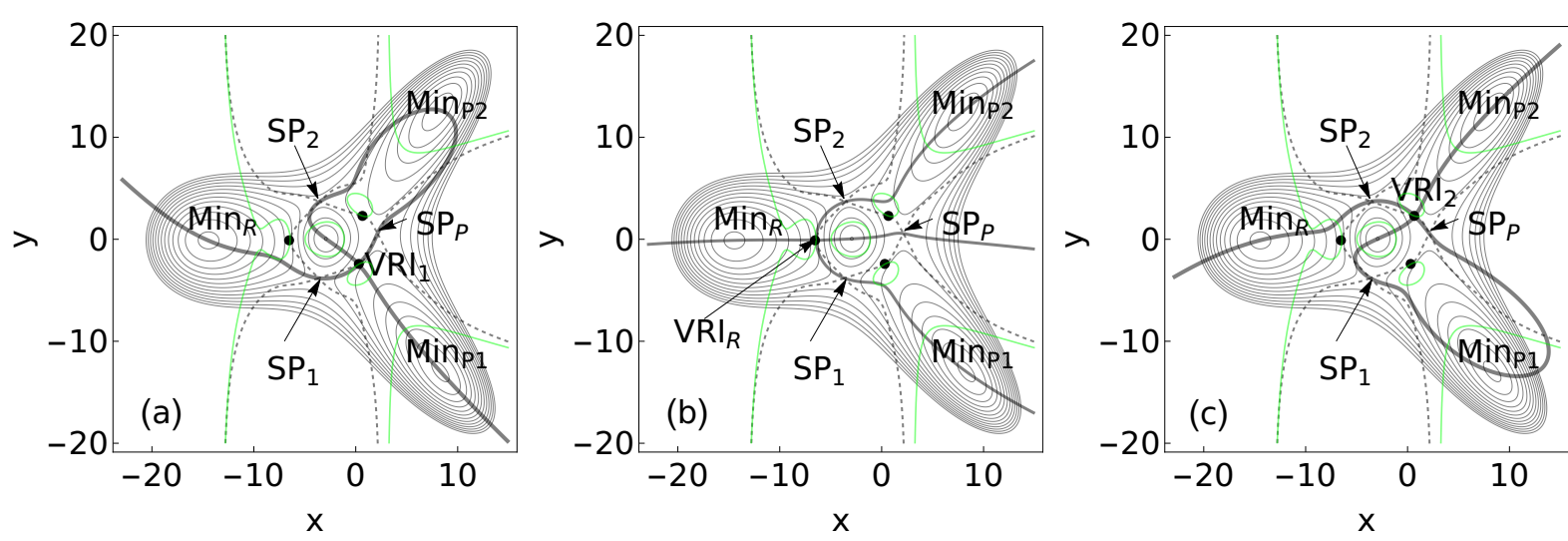

Figure 21: Three singular NTs on the modified BQC surface, see E.(4) in SI. They are the NTs which cross VRI points. They connect a respective VRI point with all stationary points of the surface. (a) NT through $\mathrm{VRI}_{1}$, (b) NT through $\mathrm{VRI}_{R}$, (c) NT through $\mathrm{VRI}_{2}$.

shown in Figure 22. Panel (a) shows the valley over $\mathrm{SP}_{1}$, but panel (b) shows the valley over $\mathrm{SP}_{2}$ The corridor between the two product minimums is discussed later. Note that every of the NTs which define these corridors are divided into two branches. One branch describes the FDSPs in the reactant valley of interest. The other branch is a loop in the contrary product valley. It describes the behaviour of the contrary SP and the contrary product minimum under a respective pulling. The contrary SP moves to the maximum and its energy increases, what is discussed elsewhere. ${ }^{10}$

Again, here emerges another type of corridor, the type 3, for NT directions outside the valley corridors. The first one is the corridor between the $\mathrm{VRI}_{1}$ right below, and a $\mathrm{VRI}_{4}$ at the top corner of the shown region, see Figure 23 (a). Contrary to the former PES example (Rhee-Pande PES), the corridor is only at the beginning a type 3 pathway (for example, from $\mathrm{R}$ to $\mathrm{SP}_{1}$, then over the maximum to $\mathrm{SP}_{2}$, but then down to $\mathrm{P}_{2}$. Thus, it goes not directly from $\mathrm{SP}_{2}$ to the aim, the $\mathrm{P}_{1}$ minimum. However, the minimum $\mathrm{P}_{2}$ is at the same time enforced by a pulling in a respective direction to $\mathrm{P}_{1}$ because the pathway $\mathrm{P}_{2}$ to $\mathrm{P}_{1}$ is an additional continuation here, a valley corridor of type 1 . So to say a type mixture happens between the type 3 corridor for the pulling of the reactant, and a type 1 corridor for an additional pulling of a possible state at $\mathrm{P}_{2}$ to the aim $\mathrm{P}_{1}$. The enforced reaction would go from $\mathrm{R}$ over an effective $\mathrm{SP}_{1}$, but also a molecule at state $\mathrm{P}_{2}$ would be enforced to react to $\mathrm{P}_{1}$. Note the spread of NTs over a wide region after $\mathrm{SP}_{2}$ in the panel (a) of Figure 23, or the spread of NTs after $\mathrm{SP}_{1}$ in the panel (b) of Figure 23. Again, an intrinsic hysteresis emerges 

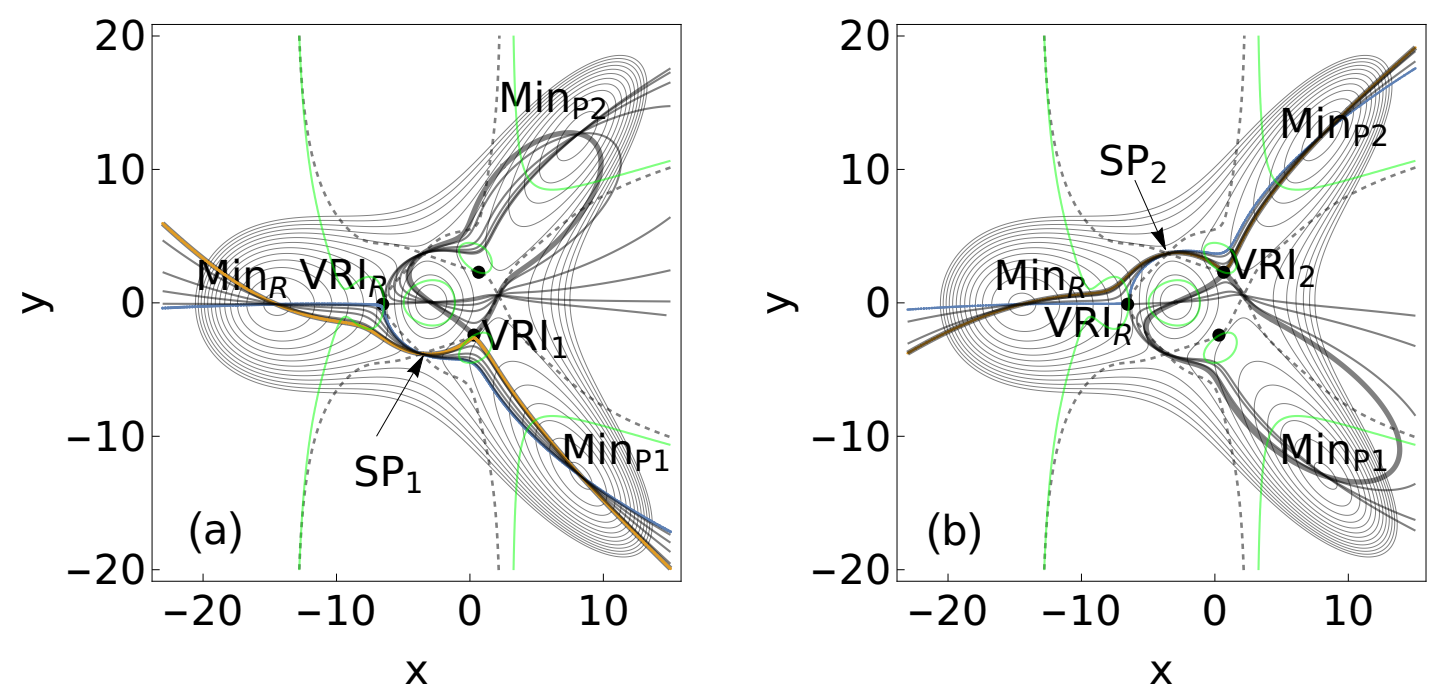

Figure 22: Two direct corridors on the modified BQC surface. (a) reaction valley between $\mathrm{VRI}_{1}$ and $\mathrm{VRI}_{R}$ over $\mathrm{SP}_{1}$, (b) between $\mathrm{VRI}_{2}$ and $\mathrm{VRI}_{R}$ over $\mathrm{SP}_{2}$.

for the reversion. An inverse direction of the force would turn the $\mathrm{P}_{1}$ to $\mathrm{P}_{2}$ by a decrease of the difference between $\mathrm{P}_{1}$ and $\mathrm{SP}_{p}$, but at the same time also the $\mathrm{SP}_{2}$ back to the product, $\mathrm{P}_{2}$. The back reaction then would be enforced over a new effective $\mathrm{SP}_{2}$ directly to the reactant, $\mathrm{R}$. The last step would be accompanied by a move together of $\mathrm{SP}_{1}$ and maximum thus closing this col. ${ }^{10}$

The last type of corridor is here a roundabout one, see Figure 24. It is of type 2. Its respective NTs are composed from two parts. The loops are now the interesting branches in the reactant valley. The other branches connect the two product minimums. If a pulling in such a direction is done, say to the $\mathrm{SP}_{1}$, then this $\mathrm{SP}_{1}$ and $\mathrm{R}$ coalesce, at least, what enforces the reaction to product $\mathrm{P}_{1}$. The $\mathrm{SP}_{2}$ and the maximum move together, at the same time. The inverse direction turns the situation and interchanges the respective stationary points. Note that many NTs in this corridor have a TP. It means that they belong usually not to the optimal region of the pathways to the respective SP. ${ }^{9,11}$ From point of view of a backreaction, $\mathrm{P}_{1} \rightarrow \mathrm{R}$, or $\mathrm{P}_{2} \rightarrow \mathrm{R}$, the directions of the corridor are forbitten. 

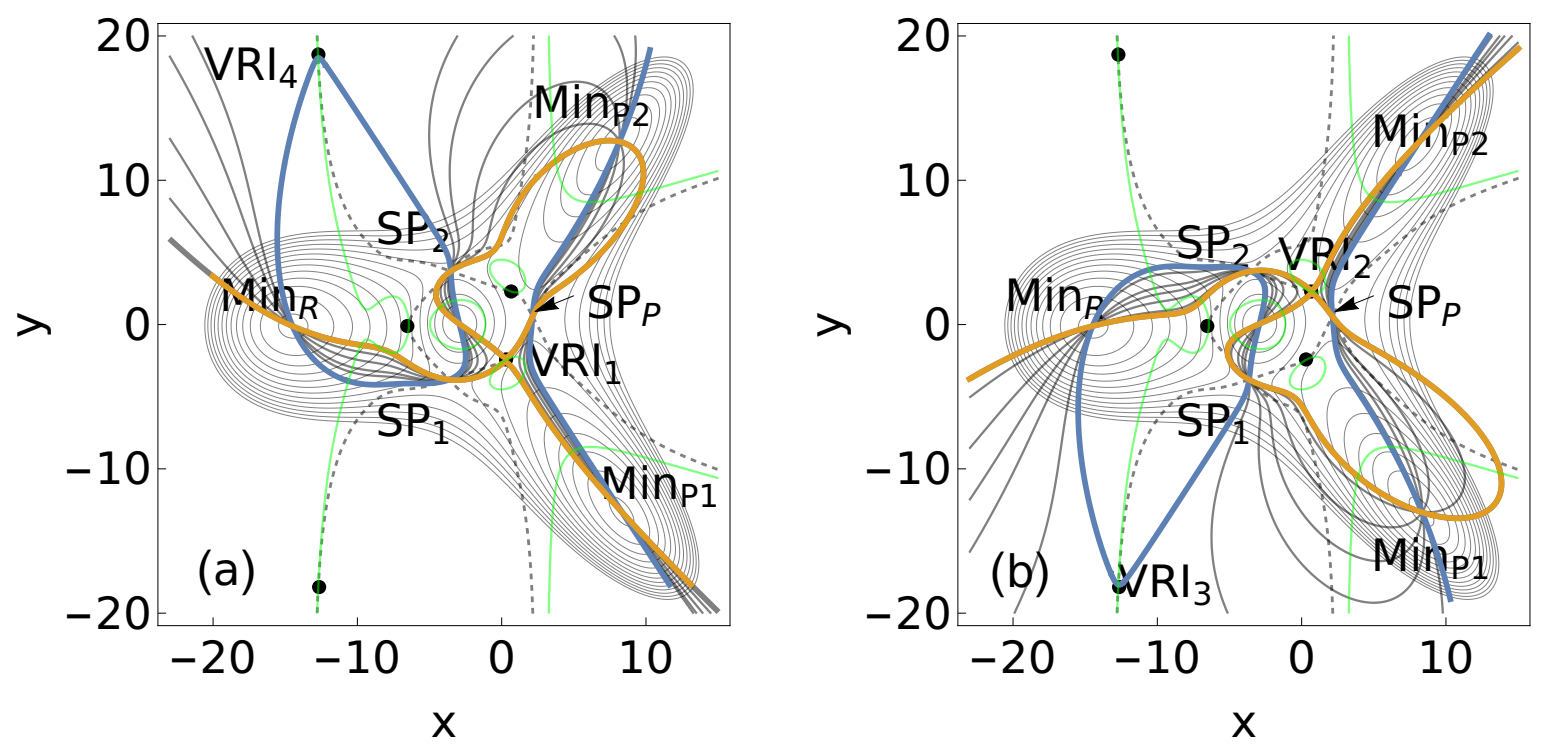

Figure 23: Corridors of type 3 on the modified BQC surface. (a) Between $\mathrm{VRI}_{1}$ and $\mathrm{VRI}_{4}$ for a pulling from $\mathrm{R}$ to $\mathrm{SP}_{1}$, (b) Between $\mathrm{VRI}_{2}$ and $\mathrm{VRI}_{3}$ for a pulling from $\mathrm{R}$ to $\mathrm{SP}_{2}$. The surface without load is shown in the background for comparison.

\section{DISCUSSION OF FURTHER EXAMPLES}

If only one SP exists between two minimums, (a very simple case which may be restricted to small molecular systems) e.g., a large set of pericyclic reactions, then there is a corridor of possible pulling directions for an enforced reaction, which is described elsewhere. ${ }^{11}$ The corridor may usually cover directions over a broad range.

For two SPs thus two competing RPs there may also be corridors of type 1. A chemical example of a type 1 corridor may be that reported by Shaik et al. ${ }^{35,36}$ in the study of the selectivity control of enzymatic-like bond activation by an external electric field. Two different oxidation pathways were considered: an oxidation over a $>\mathbf{C}=\mathbf{C}<$ double bond, epoxidation, and an oxidation over a $\mathrm{C}-\mathrm{H}$ bond, hydroxylation. When these reactions take place on the PES of a doublet state, they occur without intermediates. On the contrary, when they take place on the PES of a quartet state, an intermediate appears.

According to the study, ${ }^{35}$ the application of an external electric field to an enzymatic-like bond activation for the epoxidation of propene in its quartic electronic state, $2 \mathrm{~S}+1=4$, with the 


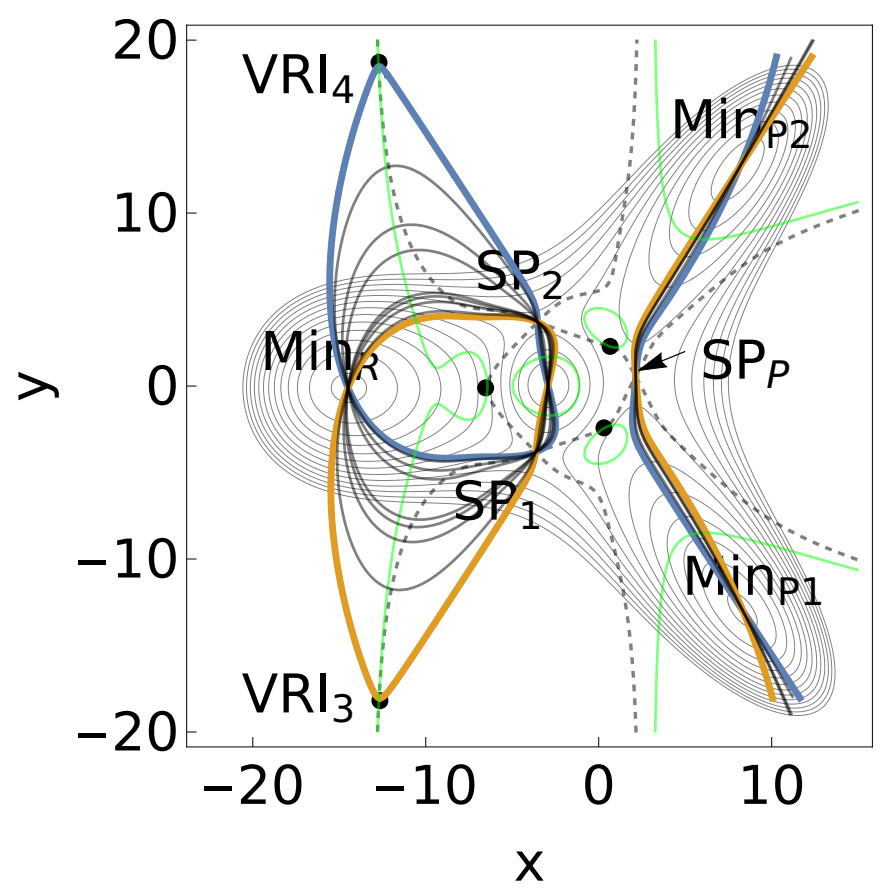

Figure 24: Roundabout corridor of type 2 on the modified BQC surface, see E.(4) in SI. Its border points are the outer VRI points 3 and 4 . Its loops connect the reactant with the both $\mathrm{SP}_{1}$ and $\mathrm{SP}_{2}$.

corresponding spin density depends on the direction of this field. In one special direction, the field leads indeed to two inverse cases of a TSS with a $\Delta E^{\ddagger}$ difference of $-1.3 \mathrm{kcal} \mathrm{mol}^{-1}$, and $-2.0 \mathrm{kcal}$ $\mathrm{mol}^{-1}$ in another direction with an applied force in both special directions of $F= \pm 0.01$ au. ${ }^{35}$ This field effect could correspond to a corridor of type 1 .

When the electronic state of the system is a doublet, $(2 \mathrm{~S}+1=2)$, with the corresponding spin density, the application of the external electric field breaks the behavior of a type 1 chemical corridor, as we will explained below.

A second reaction of propene to hydroxylation, again in both of the spin states 2 or 4 , does also not show the corridor type 1 behavior because the energy can increase after the TS. ${ }^{35}$

Possibly a simple PES model can explain the results of Shaik et al, ${ }^{35}$ see Figure 25 . The figure shows the PES without load, and some NTs for the possible pathways of the FDSPs. The formula is given in the Supplementary Information, Eq.(S4). The way to the product $\mathrm{P}_{1}$ may be the epoxidation, but the way to the product $\mathrm{P}_{2}$ may be the hydroxylation, of propene. The spin state is that of a doublet, $(2 \mathrm{~S}+1=2)$. In this case no intermediates emerge. The force to pull (in whatever 
$x$-direction ${ }^{35}$ ) along a certain NT on $\mathrm{SP}_{1}$ leads to a $\Delta E^{\ddagger}=0$ for field strength +0.01 au, but a TSS for the case $-0.01 \mathrm{au}$ by $\Delta E^{\ddagger}=-3.5 \mathrm{kcal} \mathrm{mol}^{-1}$. We guess that the NT-pathway for the effective SP moves here only for the negative field downward. For the positive field the effective SP moves along a level line. That is possible in a type 2 corridor, at least. If one looks for the $\mathrm{SP}_{1}$ on the figure than there NTs emerge of a corridor of type 2 which go along the hight of the SP. (Note that the $x$ axis in Shaik's work corresponds in the present PES Figure 25 more to the diagonal axis joining the top-left vertex and the right-bottom vertex.)

The other enforced reaction of the propene is hydroxylation ${ }^{35}$ represented by $\mathrm{SP}_{2}$ and $\mathrm{Min}_{P 2}$. For the spin state 2 one gets a similar, but inverse behavior as above at $\mathrm{SP}_{2}: \Delta E^{\ddagger}=0$ for field strength $-0.01 \mathrm{au}$ but $\Delta E^{\ddagger}=-8.4 \mathrm{kcal} \mathrm{mol}^{-1}$ for field $+0.01 \mathrm{au}$. This inverse behavior is dictated because we guess that we are on the competing SPs of the PES. There the pulling along NTs can act in an inverse kind, compare Figure 23. If one looks for the $\mathrm{SP}_{2}$ on the figure than there NTs emerge of a corridor type 2 which also go along the hight of the SP.

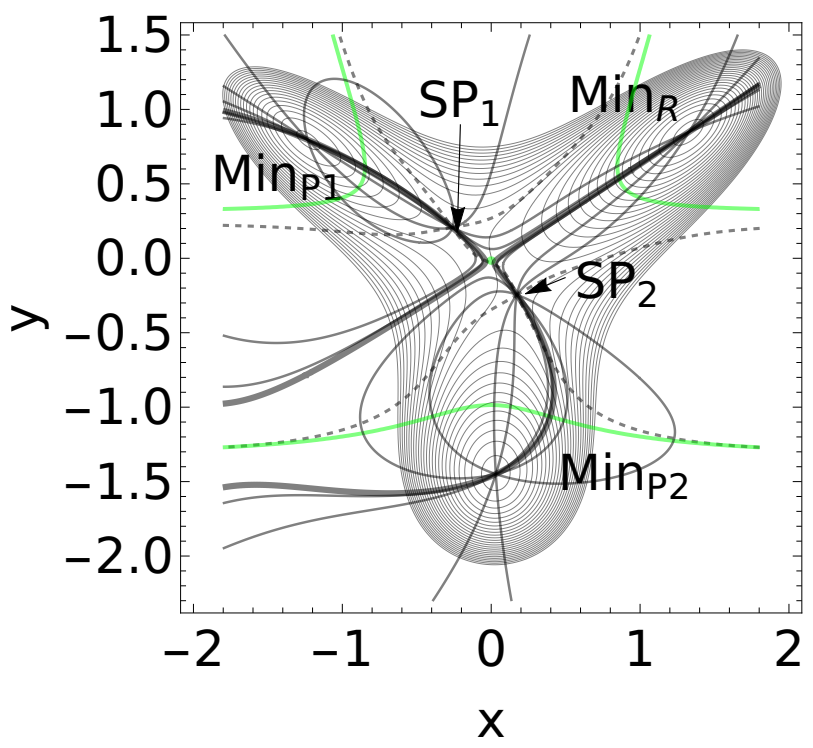

Figure 25: A second modified BQC surface with three minimums and two SPs, as well as some NTs. It may be a pattern for a propene reaction, see text. The green lines correspond to the BBP condition $\operatorname{Det}(\mathbf{H})=0$. The dashed line is the convexity border of the PES. The fat NT is the singular NT through a VRI point. Some NTs are of type 2.

By the way, not shown in the figure is the spin state 4. Still more of type 3 seems to be an 
electric pulling for the spin state 4 with $\Delta E^{\ddagger}=2.7 \mathrm{kcal} \mathrm{mol}^{-1}$ for field -0.01 au but $\Delta E^{\ddagger}=-6.3 \mathrm{kcal}$ $\mathrm{mol}^{-1}$ for field $+0.01 \mathrm{au}$. The FDSPs show the starting behaviour of NTs of a type 3 corridor, at least.

We find the same behavior in two examples of Ref. 76. There theoretical calculations of the application of an electric field are done for butadiene and ethylene, and for maleic anhydride and cyclopentadiene. The field aligned in an appropriate direction leads to a nice lowering of $\Delta E^{\ddagger}, \mathrm{a}$ TSS case. However, again the other direction leads to a higher effective $\Delta E^{\ddagger}$. The authors discuss such a behavior with electronic properties of the molecule; however one reason may be simply the shape of the respective NTs in case of a pulling along a chemical corridor of type 2,3 , or 4 .

Still more complicated are molecular motors: they have two different acting forces. Firstly there is the electrostatic force, $\mathbf{f}_{1}$, of the enzyme itself, ${ }^{26,111}$ and secondly there is a mechanical force, $\mathbf{f}_{2}$, acting by a quite large and complicated machinery. ${ }^{112}$ Already in 2001, Bustamante et al. wrote: "A motor requires specific interaction between its parts and a catalytic interaction with its fuel molecule". ${ }^{113}$ Because molecular motors are real, we see that nature has done a wide play for combining such two force vectors successfully. And now, mankind has started to do it also. ${ }^{114,115}$

\section{REMARKS AND CONCLUSIONS}

The corridor of an interesting pulling or pushing is a highly searched subject in mechanochemistry, as we can see, e.g., in Ref. 116. There the pullings of different 1,2,3-triazoles are tested by trial and error: some of the tested directions of a cyclorreversion are successful, many others not. A more theoretical way would be to locate the optimal BBP of the corresponding PES, and possibly the border VRIs. They give the possible directions of a promising pulling or pushing. It is clear that a detailed knowledge of the full corridor of possibilities provides most useful information that can be exploited in experiments. 
A technical possible pulling may have some further restrictions. Note that the authors of Ref. 116 only tested pullings between two anchor atoms: That means they use force vectors of the restricted form $\mathbf{f}_{k}=\left(0, \ldots, 0, f_{k, i}, 0, \ldots 0, f_{k, j}, 0 \ldots 0\right)^{T}$ with two times three components $f_{k, i}=-f_{k, j} \neq$ 0 for the Cartesian coordinates, $k=1,2,3$, of the anchor atoms, $i, j$. A similar ansatz is used elsewhere. ${ }^{117}$ Usually, a pulling could use a more filled vector, f, for example for a full force vector of an electrical field. ${ }^{29}$ If the structure of the catalytic side is known, one can determine the direction of the electrostatic force, see for example the Figure 2 in Ref. 118.

The use of Cartesian coordinates in the given example, of Ref. 116, should not mean that we undergo in our theory a coordinate transformation. In contrast, the Eq.(1) can be simply applied in the form of Cartesians; the $N$ former $r_{i}$ coordinates become simply $N+6$ coordinates $x_{j}$ with $j=1, \ldots, N+6$. It is another, again a linear ansatz. However, note: the theory of NTs can be used independent on the coordinate system. ${ }^{119}$

Note that there is an application of a non-linear ansatz of a force vector, instead of Eq.(1), the Artificial Force Induced Reaction (AFIR) method. ${ }^{37-39}$ However, the version is used only for numerical reasons to calculate transition states. It is described as a method without a physical meaning. Some phenomenons there observed are similar to our results.

This article has not given a full proof for the applicability of the used models to mechanochemical or biochemical processes. It proposes to deepen an already discussed model, Eq. (1), by some better mathematical curves, the NTs, which are to use for the curve of the force displaced stationary points, FDSPs. Armed with the knowledge of the mathematical structure of NTs, and if we use the simplest possible ansatz, Eq. (1), the linear one, then we can predict the transition state stabilization (TSS) by NTs, or respectively also a destabilization. We use here only two-dimensional, minimal parsimonious models with two or three minimums, and with one, two, or three SPs. However, we can propose a new, very interesting behavior of the curve of FDSPs on different kinds of energy surfaces. 
In order to find the optimal force to be applied to a molecular system for a particular rearrangement it is crucial to know the direction of this force. Previous works by us demonstrated that this optimal direction can be rigorously and elegantly found using the Newton Trajectory model and Catastrophe theory. Drawing on this theoretical framework we present here a complete and rigorous classification of different effects resulting from different force directions (the so-called 'pulling corridors'). This classification spans many possible phenomena or cases so far reported in the field of mechanochemistry and electric field induced reactions (catch-bonds, chemical hysteresis phenomena, enzymatic reaction enhanced by external electric field, mechanophores, molecular motors, etc...).

We summarize this study with some remarks:

1. The results are of topological nature as mentioned in the Introduction section and other parts of the article. Thus the reasoning is general and it is not based on the type of calculation used to obtain the PES or the free energy surface.

2. According to the results previously exposed, the mechanochemistry and the enzymatic catalysis (or inhibition) can be seen as catastrophes (transitions) of the original reaction to the modified reaction. This is based on the topological theory of Catastrophes. ${ }^{69-71}$ Note that the manifold $\operatorname{Det}(\mathbf{H})=0$ is crucial in the present context and determines the type of catastrophe. ${ }^{10}$

3. The previous theory is variational in nature or optimal control theory. One can see that the vector, $\mathbf{l}$, controls the optimal push or pull of the process. This control vector is associated to the optimal BBP, ${ }^{10}$ which is a point of an optimal NT. The nature of this curve is variational, see Ref. 8. The NT curve is the background of the present study.

4. The optimal BBP and the associated $\mathbf{l}$-vector give all the information needed for pullingpushing mechanochemistry or for a construction of an optimal catalyst (enzyme) for a reaction. Taking this information and a set of libraries about materials or aminoacids one 
can build by dynamic programing the optimal catalyst (inhibitor) or enzyme for the reaction under consideration. This is the next future.

5. For enzymes holds: if Eq. (1) is a valid model for the intrinsic structure of the enzyme activity, then we can slightly change the early view of Halder ${ }^{40}$ and Pauling ${ }^{41}$ that the bulk of the usual very big enzymes exists to maintaining the active site in a geometry faithful to the transition state. ${ }^{120}$ We now introduce the hypothesis that the bulk of the enzyme causes the active site developing an optimal force vector for the substrate. The enzyme is optimal for the best possible movement of the effective minimum and of the effective SP together on the free energy surface.

Newton trajectories (NT) can be used on the original PES or free energy surface without a load for a model of the path following 'force displaced stationary points' (FDSPs) of every developed effective PES under a pulling or pushing force, or under the action of the electrostatic force of a catalytic environment of a molecule. We continuously assume that the model Eq. (1) is in accordance with the experiment of interest. Especially, the kind of curves of Newton trajectories forms an important model for the treatment of mechanochemistry or catalysis. The model Eq. (1) realizes by a simple formula the changes of a force into changes in the kinetics of a reaction. Here we develop a model for corridors of NTs which describe the pulling possibility of direct and direct inverse pullings between two minimums (usually small corridors), or corridors with an intrinsic hysteresis between forward and backward directions over competing reaction pathways (usually breight corridors), or circular corridors which may enforce only one (forward) reaction direction, but not the inverse one.

We do believe that the classification reported and deeply analyzed in this paper provides a new language and new rules that will be employed in future to rationalize mechanochemical and enzyme phenomena.

We end this article paraphrasing Carpenter et al., ${ }^{121}$ "It is also irresistible to comment that experimental test of the predictions in the present work would be most welcome!" 


\section{SUPPORTING INFORMATION AVAILABLE}

The mathematical formulas of the diverse PES of the paper are available in the Supporting Information. This material is to get free of charge via the Internet at http://pubs.acs.org.

\section{ACKNOWLEDGMENT}

Financial support from the Spanish Ministerio de Economía y Competitividad, Project CTQ201676423-P, is acknowledged. We give thanks to Mr. Sergio Pablo García Carrillo for assistance in preparing some graphical material.

\section{References}

(1) Quapp, W.; Hirsch, M.; Imig, O.; Heidrich, D. Searching for Saddle Points of Potential Energy Surfaces by Following a Reduced Gradient. J. Comput. Chem. 1998, 19, 1087-1100.

(2) Quapp, W.; Hirsch, M.; Heidrich, D. Bifurcation of Reaction Pathways: the Set of Valley Ridge Inflection Points of a Simple Three-dimensional Potential Energy Surface. Theor. Chem. Acc. 1998, 100, 285-299.

(3) Anglada, J. M.; Besalú, E.; Bofill, J. M.; Crehuet, R. On the Quadratic Reaction Path Evaluated in a Reduced Potential Energy Surface Model and the Problem to Locate Transition States. J. Comput. Chem. 2001, 22, 387-406.

(4) Bofill, J. M.; Anglada, J. M. Finding Transition States using Reduced Potential Energy Surfaces. Theor. Chem. Acc. 2001, 105, 463-472.

(5) Crehuet, R.; Bofill, J. M.; Anglada, J. M. A new Look at the Reduced-gradient-following Path. Theor. Chem. Acc. 2002, 107, 130-139.

(6) Quapp, W. Reduced Gradient Methods and their Relation to Reaction Paths. J. Theoret. Comput. Chem. 2003, 2, 385-417. 
(7) Quapp, W. How Does a Reaction Path Branching Take Place? A Classification of Bifurcation Events. J. Molec. Struct. 2004, 695-696, 95-101.

(8) Bofill, J. M.; Quapp, W. Variational Nature, Integration, and Properties of the Newton Reaction Path. J. Chem. Phys. 2011, 134, 074101.

(9) Quapp, W.; Bofill, J. M. Pathways of most Mechanochemical Transformations are Newton Trajectories. J. Phys. Chem. B 2016, 120, 2644 - 2645.

(10) Quapp, W.; Bofill, J. M. A Contribution to a Theory of Mechanochemical Pathways by Means of Newton Trajectories. Theoret. Chem. Acc. 2016, 135, 113.

(11) Quapp, W.; Bofill, J. M. Reaction Rates in a Theory of Mechanochemical Pathways. J. Comput. Chem. 2016, 37, 2467 - 2478.

(12) Beyer, M. K.; Clausen-Schaumann, H. Mechanochemistry: The Mechanical Activation of Covalent Bonds. Chem. Rev. 2005, 105, 2921-2948.

(13) Caruso, M. M.; Davis, D. A.; Shen, Q.; Odom, S. A.; Sottos, N. R.; White, S. R.; Moore, J. S. Mechanically-Induced Chemical Changes in Polymeric Materials. Chem. Rev. 2009, 109, 5755-5798.

(14) Kucharski, T. J.; Boulatov, R. The Physical Chemistry of Mechanoresponsive Polymers. J. Mater. Chem. 2011, 21, $8237-8255$.

(15) Ribas-Ariño, J.; Marx, D. Covalent Mechanochemistry: Theoretical Concepts and Computational Tools with Applications to Molecular Nanomechanics. Chem. Rev. 2012, 112, $5412-5487$.

(16) Baláž, P.; Achimovičová, M.; Baláž, M.; Billik, P.; Cherkezova-Zheleva, Z.; Criado, J. M.; Delogu, F.; Dutková, F.; Gaffet. E.; Gotor, F. J.; et al. Hallmarks of Mechanochemistry: from Nanoparticles to Technology. Chem. Soc. Rev. 2013, 42, 7571-7637. 
(17) Wang, J.; Kouznetsova, T. B.; Niu, Z.; Ong, M. T.; Klukovich, H. M.; Rheingold, A. L.; Martinez, T. J.; Craig, S. L. Inducing and Quantifying Forbidden Reactivity with SingleMolecule Polymer Mechanochemistry. Nat. Chem. 2015, 7, 323-327.

(18) Makarov, D. E. Single Molecule Science: Physical Principles and Models; CRC Press, Taylor \& Francis Group: Boca Raton, 2015; p 214.

(19) Makarov, D. E. Perspective: Mechanochemistry of Biological and Synthetic Molecules. J. Chem. Phys. 2016, 144, 030901.

(20) Subramanian, G.; Mathew, N.; Leiding, J. A generalized Force-modified Potential Energy Surface for Mechanochemical Simulations. J.Chem.Phys. 2015, 143, 134109.

(21) Jha, S. K.; Brown, K.; Todde, G.; Subramanian, G. A Mechanochemical Study of the Effect of Compression on a Diels-Alder Reaction. J.Chem.Phys. 2016, 145, 074307.

(22) Zhuravlev, P. I.; Hinczewski, M.; Chakrabarti, S.; Marqusee, S.; Thirumalai, D. Forcedependent Switch in Protein Unfolding Pathways and Transition State Movements. Proc. Natl. Acad. Sci. 2016, 113, E715 - E724.

(23) Stauch, T.; Dreuw, A. Advances in Quantum Mechanochemistry: Electronic Structure Methods and Force Analysis. Chem. Rev. 2016, 116, 14137-14180.

(24) Eying, H.; Walter, J.; Rimball, G. E. Quantum Chemistry; John Wiley and Sons, Inc., New York, 1944.

(25) Bell, G. I. Models for the Specific Adhesion of Cells to Cells. Science 1978, 200, 618 - 627.

(26) Bustamente, C.; Chemla, Y. R.; Forde, N. R.; Izhaky, D. Mechanical processes in Biochemistry. Ann. Rev. Biochem. 2004, 73, $705-748$.

(27) Ong, M. T.; Leiding, J.; Tao, H.; Virshup, A. M.; Martínez, T. J. First Principles Dynamics and Minimum Energy Pathways for Mechanochemical Ring Opening of Cyclobutene. $J$. Am. Chem. Soc. 2009, 131, $6377-6379$. 
(28) Ribas-Ariño, J.; Shiga, M.; Marx, D. Understanding Covalent Mechanochemistry. Angew. Chem., Int. Ed. 2009, 48, 4190.

(29) Wolinski, K.; Baker, J. Theoretical Predictions of Enforced Structural Changes in Molecules. Molec. Phys. 2009, 107, 2403 -2417.

(30) Avdoshenko, S. M.; Makarov, D. E. Reaction Coordinates and Pathways of Mechanochemical Transformations. J. Phys. Chem. B 2015, 120, 1537 - 1545.

(31) Wang, J.; Kouznetsova, T. B.; Niu, Z.; Rheingold, A. L.; Craig, S. L. Accelerating a Mechanically Driven Anti-Woodward-Hoffmann Ring Opening with a Polymer Lever Arm Effect. J. Org. Chem. 2015, 80, 11895-11898.

(32) Wu, D.; Lenhardt, J. M.; Black, A. L.; Akhremitchev, B. B.; Craig, S. L. Molecular Stress Relief Through a Force-induced Irreversible Extension in Polymer Contour Length. J. Am. Chem. Soc. 2010, 132, 15936 - 15938.

(33) Thornton, E. R. A Simple Theory for Predicting the Effects of Substituent Changes on Transition State Geometry. J. Am. Chem. Soc. 1967, 89, 2915 - 2927.

(34) Aragonès, A. C.; Haworth, N. L.; Darwish, N.; Ciampi, S.; Bloomfield, N. J.; Wallace, G. G.; Diez-Perez, I.; Coote, M. L. Electrostatic Catalysis of a Diels-Alder Reaction. Nature 2016, 531, $88-91$.

(35) Shaik, S.; de Visser, S. P.; Kumar, D. External Electric Field will Control the Selectivity of Enzymatic-Like Bond Activations. J. Am. Chem. Soc. 2004, 126, 11746 - 11749.

(36) Shaik, S.; Mandal, D.; Ramanan, R. Oriented Electric Fields as Future Smart Reagents in Chemistry. Nat. Chem. 2016, 8, $1091-1098$.

(37) Maeda, S.; Morokuma, K. Communications: A Systematic Method for Locating Transition Structures of $\mathrm{A}+\mathrm{B} \rightarrow \mathrm{X}$ Type Reactions. J. Chem. Phys. 2010, 132, 241102. 
(38) Maeda, S.; Ohno, K.; Morokuma, K. Systematic Exploration of the Mechanism of Chemical Reactions: the Global Route Mapping (GRRM) Strategy Using ADDF and AFIR Methods. Phys. Chem. Chem. Phys. 2013, 15, 3683-3701.

(39) Sameera, W. M. C.; Sharma, A. K.; Maeda, S.; Morokuma, K. Artificial Force Induced Reaction Method for Systematic Determinatin of Complex Reaction Mechanisms. Chem. Rec. 2016, 16, 2349-2363.

(40) Haldane, J. B. S. Enzymes; Green, London, 1930.

(41) Pauling, L. Molecular Architecture and Biological Reactions. Chem. Eng. News 1946, 24, $1375-1377$.

(42) Warshel, A. Energetics of Enzyme Catalysis. Proc. Natl. Acad. Sci. U.S.A 1978, 75, 5250 5254.

(43) Schowen, R. L. In Transition States of Biochemical Processes; Gandour, R. D., Schowen, R. L., Eds.; Springer Science \& Business Media, 1978; pp 77-114.

(44) Burschowsky, D.; van Eerde, A.; Ökvist, M.; Kienhöfer, A.; Kast, P.; Hilvert, D.; Krengel, U. Electrostatic Transition State Stabilization Rather than Reactant Destabilization Provides the Chemical Basis for Efficient Chorismate Mutase Catalysis. Proc. Natl. Acad. Sci. U S A 2014, 111, 17516 - 17521.

(45) Warshel, A. Dynamics of Enzymatic Reactions. Proc. Natl. Acad. Sci. U.S.A 1984, 81, 444 $-448$.

(46) Warshel, A.; Sharma, P. K.; Kato, M.; Xiang, Y.; Liu, H.; Olsson, M. H. M. Electrostatic Basis for Enzyme Catalysis. Chem. Rev. 2006, 106, 3210 - 3235.

(47) Warshel, A.; Bora, R. P. Perspective: Defining and Quantifying the Role of Dynamics in Enzyme Catalysis. J. Chem. Phys. 2016, 144, 180901. 
(48) Piermattei, A.; Karthikeyan, S.; Sijbesma, R. P. Activating Catalysts with Mechanical Force. Nat. Chem. 2009, 1, 133 - 137.

(49) Groote, R.; Jakobs, R. T. M.; Sijbesma, R. P. Mechanocatalysis: Forcing Latent Catalysts into Action. Polymer Chem. 2013, 4, 4846 - 4859.

(50) Wiita, A. P.; Perez-Jimenez, R.; Walther, K. A.; Gräter, F.; Berne, B. J.; Holmgren, A.; Sanchez-Ruiz, J. M.; Fernandez, J. M. Probing the Chemistry of Thioredoxin Catalysis with Force. Nature 2007, 450, 124-127.

(51) Roy, M.; Grazioli, G.; Andricioaei, I. Rate Turnover in Mechano-catalytic Coupling: A Model and its Microscopic Origin. J. Chem. Phys. 2015, 143, 045105.

(52) Tian, Y.; Boulatov, R. Comparison of the Predictive Performance of the Bell-Evans, Taylorexpansion and Statistical-mechanics Models of Mechanochemistry. Chem. Commun. 2013, $49,4187-4189$.

(53) Dudko, O. K.; Hummer, G.; Szabo, A. Intrinsic Rates and Activation Free Energies from Single-molecule Pulling Experiments. Phys. Rev. Lett. 2006, 96, 108101.

(54) Best, R. B.; Paci, E.; Hummer, G.; Dudko, O. K. Pulling Direction as a Reaction Coordinate for the Mechanical Unfolding of Single Molecules. J. Phys. Chem. B 2008, 112, 5968-5976.

(55) Suzuki, Y.; Dudko, O. K. Biomolecules under Mechanical Stress: A Simple Mechanism of Complex Behavior. J. Chem. Phys. 2011, 134, 065102.

(56) Konda, S. S. M.; Brantley, J. M.; Bielawski, C. W.; Makarov, D. E. Chemical Reactions Modulated by Mechanical Stress: Extended Bell Theory. J. Chem. Phys. 2011, 135, 164103.

(57) Krupička, M.; Marx, D. Disfavoring Mechanochemical Reactions by Stress-Induced Steric Hindrance. J. Chem. Theory Comput. 2015, 11, 841 - 846. 
(58) Bailey, A.; Mosey, N. J. Prediction of Reaction Barriers and Force-induced Instabilities under Mechanochemical Conditions with an Approximate Model: A Case Study of the Ring Opening of 1,3-cyclohexadiene. J. Chem. Phys 2012, 136, 044102.

(59) Gensler, M.; Eidamshaus, C.; Galstyan, A.; Knapp, E.-W.; Reissig, H.-U.; Rabe, J. P. Mechanical Rupture of Mono- and Bivalent Transition Metal Complexes in Experiment and Theory. J. Phys. Chem. C 2015, 119, 4333 - 4343.

(60) Branin, F. H. Widely Convergent Methods for Finding Multiple Solutions of Simultaneous Nonlinear Equations. IBM J. Res. Develop. 1972, 16, 504-522.

(61) Quapp, W. Can We Understand the Branching of Reaction Valleys for more than two Degrees of Freedom? J. Math. Chem. 2015, 54, 137-148.

(62) Fukui, K. A Formulation of the Reaction Coordinate. J. Phys. Chem. 1970, 74, 4161-4163.

(63) Beyer, M. K. The Mechanical Strength of a Covalent Bond Calculated by Density Functional Theory. J. Chem. Phys. 2000, 112, 7307.

(64) Konda, S. S. M.; Avdoshenko, S. M.; Makarov, D. E. Exploring the Topography of the Stress-modified Energy Landscapes of Mechanosensitive Molecules. J. Chem. Phys 2014, $140,104114$.

(65) Brockwell, D. J. Force Denaturation of Proteins - an Unfolding Story. Curr. Nanosci. 2007, $3,3-15$.

(66) Ribas-Ariño, J.; Shiga, M.; Marx, D. Mechanochemical Transduction of Externally Applied Forces to Mechanophores. J. Am. Chem. Soc. 2010, 132, 10609 - 10614.

(67) Iozzi, M. F.; Helgaker, T.; Uggerud, E. Assessment of Theoretical Methods for the Determination of the Mechanochemical Strength of Covalent Bonds. Mol. Phys. 2009, 107, 2537.

(68) Freund, L. B. Characterizing the Resistance Generated by a Molecular Bond as it is Forcibly Separated. Proc. Nat. Acad. Sci. 2009, 106, 8818 - 8823. 
(69) Heidrich, D.; Kliesch, W.; Quapp, W. Properties of Chemically Interesting Potential Energy Surfaces; Springer: Berlin, Heidelberg, 1991.

(70) Thom, R. Structural Stability and Morphogenesis: An Outline of a General Theory of Models; Addison-Wesley: Reading, MA, 1989.

(71) Gilmore, R. Catastrophe Theory for Scientists and Engineers; Dover, New York, 1993.

(72) Hirsch, M.; Quapp, W. Reaction Pathways and Convexity of the Potential Energy Surface: Application of Newton Trajectories. J. Math. Chem. 2004, 36, 307-340.

(73) Bofill, J. M.; Quapp, W. The Variational Nature of the Gentlest Ascent Dynamics and the Relation of a Variational Minimum of a Curve and the Minimum Energy Path. Theor. Chem. Acc. 2016, 135, 11-24.

(74) Yin, J.; Andryski, S. E.; Beuscher, A. E.; Stevens, R. C.; Schultz, P. G. Structural Evidence for Substrate Strain in Antibody Catalysis. Proc. Natl. Am. Soc. 2003, 100, 856 - 861.

(75) Shaik, S. S. What Happens to Molecules as They React? A Valence Bond Approach to Reactivity. J. Am. Chem. Soc. 1981, 103, 3692 - 3701.

(76) Meir, R.; Chen, H.; Lai, W.; Shaik, S. Oriented Electric Fields Accelerate Diels-Alder Reactions and Control the Endo/Exo Selectivity. ChemPhysChem 2010, 11, $301-310$.

(77) Jiao, H.; v. R. Schleyer, P. Electrostatic Acceleration of the 1,5-H Shifts in Cyclopentadiene and in Penta-1,3-diene by $\mathrm{Li}^{+}$Complexation: Aromaticity of the Transition Structures. $J$. Chem. Soc. Faraday Trans. 1994, 90, 1559 - 1567.

(78) Röhrig, U. F.; Frank, I. First-principles Molecular Dynamics Study of a Polymer under Tensile Stress. J. Chem. Phys. 2001, 115, 8670-8674.

(79) Ribas-Ariño, J.; Shiga, M.; Marx, D. Unravelling the Mechanism of Force-Induced RingOpening of Benzocyclobutenes. Chem. Eur. J. 2009, 15, 13331-13335. 
(80) Heidrich, D.; Quapp, W. Saddle Points of Index 2 on PES and their Role in Theoretical Reactivity Investigations. Theor. Chim. Acta 1986, 70, 89-98.

(81) Ezra, G. S.; Wiggins, S. Phase-space Geometry and Reaction Dynamics near Index 2 Saddles. J. Phys. A 2009, 42, 205101.

(82) Quapp, W.; Bofill, J. M. Embedding of the Saddle Point of Index Two on the PES of the Ring Opening of Cyclobutene. Int. J. Quantum Chem. 2015, 115, 1635-1649.

(83) Llunell, M.; Alemany, P.; Bofill, J. M. Conformational Analysis of Molecular Machines: Internal Rotation and Enantiomerization in Triptycyl[3]helicene. Chem. Phys. Chem 2008, $9,1117-1119$.

(84) Sarangapani, K. K.; Yago, T.; Klopocki, A. G.; Lawrence, M. B.; Fieger, C. B.; Rosen, S. D.; McEver, R. P.; Zhu, C. Low Force Decelerates L-selectin Dissociation from P-selectin Glycoprotein Ligand-1 and Endoglycan. J. Biol. Chem. 2004, 279, 2291 - 2298.

(85) Evans, E.; Leung, A.; Volkmar, H.; Zhu, C. Mechanical Switching and Coupling between two Dissociation Pathways in a P-selectin Adhesion Bond. Proc. Natl. Acad. Sci. 2004, 101, $11281-11286$.

(86) Barsegov, V.; Thirumalai, D. Dynamics of Unbinding of Cell Adhesion Molecules: Transition from Catch to Slip bonds. Proc. Natl. Acad. Sci. 2005, 102, 1835 - 1839.

(87) Zhu, C.; Lou, J.; McEver, R. P. Catch Bonds: Physical Models, Structural Bases, Biological Function and Rheological Relevance. Biorheology 2005, 42, 443 - 462.

(88) Zhu, C.; McEver, R. P. Catch Bonds: Physical Models and Biological Functions. Molec. Cell. Biomech. 2005, 2, 91 - 104.

(89) Pereverzev, Y. V.; Prezhdo, O. V.; Thomas, W. E.; Sokurenko, E. V. Distinctive Features of the Biological Catch Bond in the Jump-ramp Force Regime Predicted by the Two-pathway Model. Phys. Rev. E 2005, 72, 010903. 
(90) Pereverzev, Y. V.; Prezhdo, O. V.; Forero, M.; Sokurenko, E. V.; Thomas, W. E. The Twopathway Model for the Catch-Slip Transition in Biological Adhesion. Biophys. J. 2005, 89, $1446-1454$.

(91) Thomas, W. E. Understanding the Counterintuitive Phenomenon of Catch Bonds. Curr. Nanosci. 2007, 3, $63-77$.

(92) Thomas, W. E.; Vogel, V.; Sokurenko, E. Biophysics of Catch Bonds. Annu. Rev. Biophys. 2008, 37, $399-416$.

(93) Prezhdo, O. V.; Pereverzev, Y. V. Theoretical Aspects of the Biological Catch Bond. Acc. Chem. Res. 2009, 42, $693-703$.

(94) Pereverzev, Y. V.; Prezhdo, E.; Sokurenko, E. V. The Two-Pathway Model of the Biological Catch-Bond as a Limit of the Allosteric Model. Biophys. J. 2011, 101, 2026 - 2036.

(95) Jagannathan, B.; Elms, P. J.; Bustamente, C.; Marqusee, S. Direct Observation of a Forceinduced Switch in the Anisotropic Mechanical Unfolding Pathway of a Protein. Proc. Nat. Acad. Sci. 2012, 109, 17820 - 17825.

(96) Kreuzer, S. M.; Moon, T. J.; Elber, R. Catch Bond-like Kinetics of Helix Cracking: Network Analysis by Molecular Dynamics and Milestoning. J. Chem. Phys. 2013, 139, 121902.

(97) Konda, S. S. M.; Brantley, J. M.; Varghese, B. T.; Wiggins, K. M.; Bielawski, C. W.; Makarov, D. E. Molecular Catch Bonds and the Anti-Hammond Effect in Polymer Mechanochemistry. J. Am. Chem. Soc. 2013, 135, 12722 - 12729.

(98) Eckhardt, B. Irregular scattering. Physica D 1988, 33, 89-98.

(99) Hirsch, M.; Quapp, W. Reaction Channels of the Potential Energy Surface: Application of Newton Trajectories. J. Molec. Struct., THEOCHEM 2004, 683, 1-13. 
(100) Tan, E. M. M.; Amirjalayer, S.; Smolarek, S.; Vdovin, A.; Zerbetto, F.; Buma, W. J. Fast Photodynamics of Azobenzene Probed by Scanning Excited-state Potential Energy Surfaces Using slow Spectroscopy. Nat.Commun. 2015, 6, 5860.

(101) Turanský, R.; Konôpka, M.; Doltsinis, N. L.; Štich, I.; Marx, D. Switching of Functionalized Azobenzene Suspended between Gold Tips by Mechanochemical, Photochemical, and Opto-mechanical Means. Phys.Chem.Chem.Phys. 2010, 12, 13922-13932.

(102) Rhee, Y. M.; Pande, V. S. One-Dimensional Reaction Coordinate and the Corresponding Potential of Mean Force from Commitment Probability Distribution. J. Phys. Chem. B 2005, $109,6780-6786$.

(103) Quapp, W.; Bofill, J. M.; Caballero, M. Search for Conical Intersection Points (CI) by Newton Trajectories. Chem. Phys. Lett. 2012, 541, 122-127.

(104) Anslyn, E. V.; Dougherty, D. A. Modern Physical Organic Chemistry; University Science Books, 2006.

(105) Dewar, M. J. S.; Dougherty, R. C. The PMO Theory of Organic Chemistry; Plenum, New York, 1975.

(106) Overman, L. E.; Knoll, F. M. Palladium(II) Chloride Catalyzed Cope Rearrangements of Acyclic 1,5-Dienes. J. Am. Chem. Soc. 1980, 102, 865 - 867.

(107) Dauben, W. G.; Chollet, A. Acid Catalyzed Cope Rearrangements of 2-acyl-1,5-dienes. Tetrahedron Lett. 1981, 22, 1583 - 1586.

(108) Kaldre, D.; Gleason, J. L. An Organocatalytic Cope Rearrangement. Angew. Chem. Int. Ed. 2016, 55, $11557-11561$.

(109) Gebauer, K.; Schneider, C. The First Organocatalytic Cope Rearrangement. Angew. Chem. Int. Ed. 2016, 55, 14208 - 14209. 
(110) Bofill, J. M.; Quapp, W.; Caballero, M. The Variational Structure of Gradient Extremals. J. Chem. Theory Comput. 2012, 8, 927-935.

(111) Mukherjee, S.; Bora, R. P.; Warshel, A. Torque, Chemistry and Efficiency in Molecular Motors: a Study of the Rotary-chemical Coupling in $\mathrm{F}_{1}$-ATPase. Quart. Rev. Biophys 2015, $48,395-403$.

(112) Itoh, H.; Takahashi, A.; Adachi, K.; Noji, H.; Yasuda, R.; Yoshida, M.; Kinosita, Jr., K. Mechanically driven ATP synthesis by $\mathrm{F}_{1}$-ATPase. Nature 2004, 427, 465 - 468.

(113) Bustamante, C.; Keller, D.; Oster, G. The Physics of Molecular Motors. Acc. Chem. Res. 2001, $34,412-420$.

(114) Special Issue on Molecular Machines, ChemPhysChem, Issue 12 2016, 17, 1710-1938.

(115) Ramström, O. Scientific Background on the Nobel Prize in Chemistry 2016. MOLECULAR MACHINES. 2016, $1-16$.

(116) Jacobs, M. J.; Schneider, G.; Blank, K. G. Mechanical Reversibility of Strain-Promoted Azide-Alkyne Cycloaddition Reactions. Angew. Chem.-Int. Ed. 2016, 55, 2899 - 2902.

(117) Kochhar, G. S.; Mosey, N. J. Differences in the Abilities to Mechanically Eliminate Activation Energies for Unimolecular and Bimolecular Reactions. Scientific Reports 2016, 6, 23059.

(118) Rees, D. M.; Montgomery, M. G.; Leslie, A. G. W.; Walker, J. E. Structure Evidence of a new Catalytic Intermediate in the Pathway of ATP Hydrolysis by $\mathrm{F}_{1}$-ATPase from Bovine Heart Mitochondria. Proc. Natl. Am. Soc. 2012, 109, 11139 - 11143.

(119) Quapp, W. Newton Trajectories in the Curvilinear Metric of Internal Coordinates. J. Math Chem. 2004, 36, 365-379.

(120) Britt, B. M. For Enzymes, Bigger is Better. Biophys. Chem. 1997, 69, 63 - 70. 
(121) Carpenter, B. K.; Harvey, J. N.; Glowacki, D. R. Prediction of Enhanced Solvent-induced Enantioselectivity for a Ring Opening with a Bifurcating Reaction Path. Phys. Chem. Chem. Phys. 2015, 17, $8372-8381$. 


\section{Graphical TOC Entry}

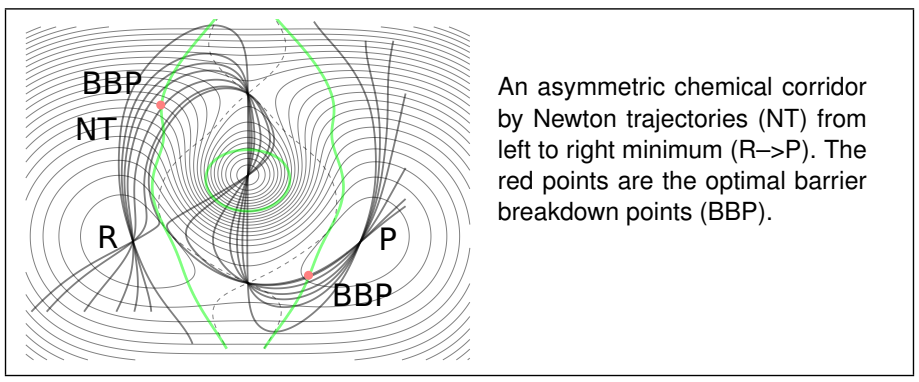

